\title{
A New Approach to Fixed Point Results in Triangular Intuitionistic Fuzzy Metric Spaces
}

\author{
N. Hussain, ${ }^{1}$ S. Khaleghizadeh, ${ }^{2}$ P. Salimi, ${ }^{2}$ and Afrah A. N. Abdou ${ }^{1}$ \\ ${ }^{1}$ Department of Mathematics, King Abdulaziz University, P.O. Box 80203, Jeddah 21589, Saudi Arabia \\ ${ }^{2}$ Department of Mathematics, Payame Noor University, P.O. Box 19395-3697, Tehran, Iran \\ Correspondence should be addressed to P. Salimi; salimipeyman@gmail.com
}

Received 25 January 2014; Revised 11 March 2014; Accepted 15 March 2014; Published 15 April 2014

Academic Editor: Ngai-Ching Wong

Copyright (c) 2014 N. Hussain et al. This is an open access article distributed under the Creative Commons Attribution License, which permits unrestricted use, distribution, and reproduction in any medium, provided the original work is properly cited.

\begin{abstract}
The aim of this paper is to propose some fixed point theorems in complete parametric metric spaces. Using these theorems, we deduce as corollaries the recent results of Ionescu et al. Moreover, we suggest some new contractions and prove certain fixed point theorems in triangular intuitionistic fuzzy metric spaces. We also discuss some illustrative examples to highlight the realized improvements.
\end{abstract}

\section{Introduction and Preliminaries}

The concept of fuzzy set was introduced by Zadeh [1] in 1965. In 1975, Kramosil and Michálek [2] introduced the notion of fuzzy metric space, which can be regarded as a generalization of the statistical (probabilistic) metric space. This work has provided an important basis for the construction of fixed point theory in fuzzy metric spaces. Afterwards, Grabiec [3] defined the completeness of the fuzzy metric space (now known as a $G$-complete fuzzy metric space) and extended the Banach contraction theorem to $G$-complete fuzzy metric spaces. Successively, George and Veeramani [4] modified the definition of the Cauchy sequence introduced by Grabiec. Meanwhile, they slightly modified the notion of a fuzzy metric space introduced by Kramosil and Michálek and then defined a Hausdorff and first countable topology on it. Since then, the notion of a complete fuzzy metric space presented by George and Veeramani has emerged as another characterization of completeness, and some fixed point theorems have also been proved on the basis of this metric space. From the above analysis, we can see that there are many studies related to fixed point theory based on the above two kinds of complete fuzzy metric spaces (see for more details [5-11] and the references therein). In 2004, Park introduced the notion of intuitionistic fuzzy metric space [12]. He showed that, for each intuitionistic fuzzy metric space $(X, M, N, *, \diamond)$, the topology generated by the intuitionistic fuzzy metric $(M, N)$ coincides with the topology generated by the fuzzy metric $M$. For more details on intuitionistic fuzzy metric space and related results we refer the reader to [12-17].

Definition 1. A 5-tuple $(X, M, N, *, \diamond)$ is said to be an intuitionistic fuzzy metric space if $X$ is an arbitrary set, * is a continuous $t$-norm, $\diamond$ is a continuous $t$-conorm, and $M, N$ are fuzzy sets on $X^{2} \times(0, \infty)$ satisfying the following conditions, for all $x, y, z \in X$ and $t, s>0$ :

(i) $M(x, y, t)+N(x, y, t) \leq 1$;

(ii) $M(x, y, t)>0$;

(iii) $M(x, y, t)=1$ for all $t>0$ if and only if $x=y$;

(iv) $M(x, y, t)=M(y, x, t)$;

(v) $M(x, y, t) * M(y, z, s) \leq M(x, z, t+s)$;

(vi) $M(x, y, \cdot):(0, \infty) \rightarrow[0,1]$ is left continuous;

(vii) $\lim _{t \rightarrow \infty} M(x, y, t)=1$;

(viii) $N(x, y, t)>0$;

(ix) $N(x, y, t)=0$ if and only if $x=y$;

(x) $N(x, y, t)=N(y, x, t)$;

(xi) $N(x, y, t) \diamond N(y, z, s) \geq N(x, z, t+s)$;

(xii) $N(x, y, \cdot):(0, \infty) \rightarrow[0,1]$ is right continuous;

(xiii) $\lim _{t \rightarrow \infty} N(x, y, t)=0$. 
Then $(M, N)$ is called an intuitionistic fuzzy metric on $X$. The functions $M(x, y, t)$ and $N(x, y, t)$ denote the degree of nearness and the degree of nonnearness between $x$ and $y$ with respect to $t$, respectively.

Definition 2. Let $(X, M, N, *, \diamond)$ be an intuitionistic fuzzy metric space. Then,

(i) a sequence $\left\{x_{n}\right\}$ is said to be Cauchy sequence whenever $\lim _{m, n \rightarrow \infty} M\left(x_{n}, x_{m}, t\right)=1$ and $\lim _{m, n \rightarrow \infty} N\left(x_{n}, x_{m}, t\right)=0$ for all $t>0$,

(ii) $(X, M, N, *, \diamond)$ is called complete whenever every Cauchy sequence is convergent with respect to the topology $\tau_{(M, N)}$.

Remark 3. Note that, if $(M, N)$ is an intuitionistic fuzzy metric on $X$ and $\left\{x_{n}\right\}$ is a sequence in $X$ such that $\lim _{m, n \rightarrow \infty} M\left(x_{n}, x_{m}, t\right)=1$, then $\lim _{m, n \rightarrow \infty} N\left(x_{n}, x_{m}, t\right)=$ 0 as, from (i) of Definition 1, we know that $M(x, y, t)+$ $N(x, y, t) \leq 1$ for all $x, y \in X$ and all $t>0$.

Definition 4 (see $[18,19])$. Let $(X, M, N, *, \diamond)$ be an intuitionistic fuzzy metric space. The fuzzy metric $(M, N)$ is called triangular whenever

$$
\begin{gathered}
\frac{1}{M(x, y, t)}-1 \leq \frac{1}{M(x, z, t)}-1+\frac{1}{M(z, y, t)}-1 \\
N(x, y, t) \leq N(x, z, t)+N(z, y, t)
\end{gathered}
$$

for all $x, y, z \in X$ and all $t>0$.

\section{From Parametric Metric to Triangular Intuitionistic Fuzzy Metric}

First we define the concept of parametric metric space.

Definition 5. Let $X$ be a nonempty set and let $\mathscr{P}: X \times X \times$ $(0, \infty) \rightarrow[0, \infty)$ be a function. We say $\mathscr{P}$ is a parametric metric on $X$ if

(i) $\mathscr{P}(x, y, t)=0$ for all $t>0$ if and only if $x=y$;

(ii) $\mathscr{P}(x, y, t)=\mathscr{P}(y, x, t)$ for all $t>0$;

(iii) $\mathscr{P}(x, y, t) \leq \mathscr{P}(x, z, t)+\mathscr{P}(z, y, t)$ for all $x, y, z \in X$ and all $t>0$.

and one says the pair $(X, \mathscr{P})$ is a parametric metric space.

Example 6. Let $X$ denote the set of all functions $f:(0, \infty) \rightarrow$ $\mathbb{R}$. Define, $\mathscr{P}: X \times X \times(0, \infty) \rightarrow[0, \infty)$ by $\mathscr{P}(f, g, t)=$ $|f(t)-g(t)|$ for all $f, g \in X$ and all $t>0$. Then $(X, \mathscr{P})$ is a parametric metric space.

Example 7. Let $(X, M, N, *, \diamond)$ be an triangular intuitionistic fuzzy metric space. We consider the mapping $\mathscr{P}: X \times X \times$ $(0, \infty) \rightarrow[0, \infty)$ defined by $\mathscr{P}(x, y, t):=1 / M(x, y, t)-1$ for all $x, y \in X$ and all $t>0$. Then by Definitions 1 (iii) and $4, \mathscr{P}$ is a parametric metric on $X$ and hence $(X, \mathscr{P})$ is a parametric metric space.
Let $(X, \mathscr{P})$ be a parametric metric space. Let $x \in X$ and $r>0$; then the set

$$
B(x, r)=\{y \in X ; \mathscr{P}(x, y, t)<r \forall t>0\}
$$

is called an open ball with center at $x$ and radius $r>0$.

Now we have the following definitions.

Definition 8. Let $(X, \mathscr{P})$ be a parametric metric space, and let $\left\{x_{n}\right\}$ be a sequence of points of $X$. A point $x \in X$ is said to be the limit of the sequence $\left\{x_{n}\right\}$, if $\lim _{n \rightarrow+\infty} \mathscr{P}\left(x, x_{n}, t\right)=0$ for all $t>0$, and one says that the sequence $\left\{x_{n}\right\}$ is convergent to $x$ and denotes it by $x_{n} \rightarrow x$ as $n \rightarrow \infty$.

Remark 9. Note that if $(X, \mathscr{P})$ is a parametric metric space and $\left\{x_{n}\right\},\left\{y_{n}\right\}$ are two sequences in $X$ such that $x_{n} \rightarrow x$ and $y_{n} \rightarrow y$ as $n \rightarrow \infty$, then $\lim _{m, n \rightarrow \infty} \mathscr{P}\left(x_{n}, y_{m}, t\right)=$ $\mathscr{P}(x, y, t)$ for all $t>0$. That is, $\mathscr{P}$ is continuous in its two variables.

Definition 10. Let $(X, \mathscr{P})$ be a parametric metric space.

(S1) A sequence $\left\{x_{n}\right\}$ is called a Cauchy if and only if $\lim _{m, n \rightarrow \infty} \mathscr{P}\left(x_{n}, x_{m}, t\right)=0$ for all $t>0$.

(S2) A parametric metric space $(X, \mathscr{P})$ is said to be complete if and only if every Cauchy sequence $\left\{x_{n}\right\}$ in $X$ converges to $x \in X$.

Definition 11. Let $(X, \mathscr{P})$ be a parametric metric space and let $T: X \rightarrow X$ be a mapping. One says $T$ is a continuous mapping at $x$ in $X$, if, for any sequence $\left\{x_{n}\right\}$ in $X$ such that $x_{n} \rightarrow x$ as $n \rightarrow \infty, T x_{n} \rightarrow$ Tx as $n \rightarrow \infty$.

Theorem 12. Let $(X, \mathscr{P})$ be a complete parametric metric space and let $T: X \rightarrow X$ be a continuous self-mapping such that

$$
\begin{aligned}
\mathscr{P}(T x, T y, t) & \\
\leq r \max & \{\mathscr{P}(x, y, t), \mathscr{P}(x, T x, t), \\
& \left.\mathscr{P}(y, T y, t), \frac{\mathscr{P}(x, T x, t) \mathscr{P}(y, T y, t)}{\mathscr{P}(x, y, t)}\right\}
\end{aligned}
$$

holds for all $x, y \in X$ with $x \neq y$ and all $t>0$ where $0 \leq r<1$. Then $T$ has a unique fixed point.

Proof. Let $x_{0} \in X$. Define the sequence $\left\{x_{n}\right\} \subset X$ by $x_{n+1}=$ $T x_{n}$ for all positive integers $n$. If there exists $n_{0} \in \mathbb{N} \cup\{0\}$ such that $x_{n_{0}}=x_{n_{0}+1}=T x_{n_{0}}$, then $x_{n_{0}}$ is fixed point of $T$ and 
we have nothing to prove. Hence we assume $x_{n} \neq x_{n+1}$ for all $n \in \mathbb{N} \cup\{0\}$. From (3) with $x=x_{n}$ and $y=x_{n-1}$ we get

$$
\begin{aligned}
& \mathscr{P}\left(x_{n+1}, x_{n}, t\right) \\
& =\mathscr{P}\left(T x_{n}, T x_{n-1}, t\right) \\
& \leq r \max \left\{\mathscr{P}\left(x_{n}, x_{n-1}, t\right), \mathscr{P}\left(x_{n}, T x_{n}, t\right)\right. \text {, } \\
& \mathscr{P}\left(x_{n-1}, T x_{n-1}, t\right), \\
& \left.\frac{\mathscr{P}\left(x_{n}, T x_{n}, t\right) \mathscr{P}\left(x_{n-1}, T x_{n-1}, t\right)}{\mathscr{P}\left(x_{n}, x_{n-1}, t\right)}\right\} \\
& \leq r \max \left\{\mathscr{P}\left(x_{n}, x_{n-1}, t\right), \mathscr{P}\left(x_{n}, x_{n+1}, t\right),\right. \\
& \mathscr{P}\left(x_{n-1}, x_{n}, t\right), \\
& \left.\frac{\mathscr{P}\left(x_{n}, x_{n+1}, t\right) \mathscr{P}\left(x_{n-1}, x_{n}, t\right)}{\mathscr{P}\left(x_{n}, x_{n-1}, t\right)}\right\} \\
& =r \max \left\{\mathscr{P}\left(x_{n}, x_{n-1}, t\right), \mathscr{P}\left(x_{n}, x_{n+1}, t\right)\right\} \text {. }
\end{aligned}
$$

Now if $\max \left\{\mathscr{P}\left(x_{n}, x_{n-1}, t\right), \mathscr{P}\left(x_{n}, x_{n+1}, t\right)\right\}=\mathscr{P}\left(x_{n}, x_{n+1}, t\right)$, then from the above inequality we get

$$
\mathscr{P}\left(x_{n+1}, x_{n}, t\right) \leq r \mathscr{P}\left(x_{n}, x_{n+1}, t\right)<\mathscr{P}\left(x_{n}, x_{n+1}, t\right)
$$

which is a contradiction. Therefore,

$$
\mathscr{P}\left(x_{n+1}, x_{n}, t\right) \leq r \mathscr{P}\left(x_{n}, x_{n-1}, t\right),
$$

for all $n \in \mathbb{N} \cup\{0\}$ and all $t>0$. Hence,

$$
\mathscr{P}\left(x_{n+1}, x_{n}, t\right) \leq r^{n} \mathscr{P}\left(x_{1}, x_{0}, t\right) .
$$

Then for any $n>m$ by (7) we have

$$
\begin{aligned}
& \mathscr{P}\left(x_{n}, x_{m}, t\right) \\
& \leq \mathscr{P}\left(x_{n}, x_{n-1}, t\right)+\mathscr{P}\left(x_{n-1}, x_{n-2}, t\right) \\
&+\cdots+\mathscr{P}\left(x_{m+1}, x_{m}, t\right) \\
& \leq\left(r^{n-1}+r^{n-2}+\cdots+r^{m}\right) \mathscr{P}\left(x_{1}, x_{0}, t\right) \\
& \leq \frac{r^{m}}{1-r} \mathscr{P}\left(x_{1}, x_{0}, t\right),
\end{aligned}
$$

for all $t>0$. By taking limit as $m, n \rightarrow \infty$ in the above inequality we get $\lim _{m, n \rightarrow \infty} \mathscr{P}\left(x_{n}, x_{m}, t\right)=0$. Therefore, $\left\{x_{n}\right\}$ is a Cauchy sequence in $X$. Since $(X, \mathscr{P})$ is a complete parametric metric space, there exists $x^{*} \in X$ such that $x_{n} \rightarrow$ $x^{*}$ as $n \rightarrow \infty$. Now since $T$ is continuous,

$$
x^{*}=\lim _{n \rightarrow \infty} x_{n+1}=\lim _{n \rightarrow \infty} T x_{n}=T x^{*} .
$$

That is, $T$ has a fixed point. To prove the uniqueness of $x^{*}$, suppose that $y^{*}$ is another fixed point of $T$ such that $x^{*} \neq y^{*}$. From (3) we obtain

$$
\begin{aligned}
& \mathscr{P}\left(x^{*}, y^{*}, t\right) \\
& =\mathscr{P}\left(T x^{*}, T y^{*}, t\right) \\
& \leq r \max \left\{\mathscr{P}\left(x^{*}, y^{*}, t\right), \mathscr{P}\left(x^{*}, T x^{*}, t\right),\right. \\
& \mathscr{P}\left(y^{*}, T y^{*}, t\right), \\
& \left.\frac{\mathscr{P}\left(x^{*}, T x^{*}, t\right) \mathscr{P}\left(y^{*}, T y^{*}, t\right)}{\mathscr{P}\left(x^{*}, y^{*}, t\right)}\right\} \\
& =r \mathscr{P}\left(x^{*}, y^{*}, t\right)<\mathscr{P}\left(x^{*}, y^{*}, t\right),
\end{aligned}
$$

which is a contradiction. So, $T$ has a unique fixed point.

Example 13. Let $X=[0, \infty)$ be endowed with the parametric metric

$$
\mathscr{P}(x, y, t)= \begin{cases}t \max \{x, y\}, & x \neq y \\ 0, & x=y,\end{cases}
$$

for all $x, y \in X$ and all $t>0$. Define $T: X \rightarrow X$ by

$$
T x= \begin{cases}\frac{1}{5} x^{2}, & \text { if } x \in[0,1) \\ \frac{1}{5} x, & \text { if } x \in[1,2) \\ \frac{2}{5} & \text { if } x \in[2, \infty) .\end{cases}
$$

Clearly, $(X, \mathscr{P})$ is a complete parametric metric space and $T$ is a continuous mapping. Now we consider the following cases:

(i) Let $x, y \in[0,1)$. Then,

$$
\begin{aligned}
& \mathscr{P}(T x, T y, t) \\
& =t \max \left\{\frac{1}{5} x^{2}, \frac{1}{5} y^{2}\right\} \leq \frac{3}{5} t \max \{x, y\} \\
& \leq \frac{3}{5} \max \{\mathscr{P}(x, y, t), \mathscr{P}(x, T x, t), \\
& \left.\mathscr{P}(y, T y, t), \frac{\mathscr{P}(x, T x, t) \mathscr{P}(y, T y, t)}{\mathscr{P}(x, y, t)}\right\} .
\end{aligned}
$$

(ii) Let $x, y \in[1,2)$. Then,

$\mathscr{P}(T x, T y, t)$

$$
=t \max \left\{\frac{1}{5} x, \frac{1}{5} y\right\} \leq \frac{3}{5} t \max \{x, y\}
$$




$$
\begin{aligned}
\leq \frac{3}{5} \max \{\mathscr{P}(x, y, t), \mathscr{P}(x, T x, t), \\
\left.\qquad \mathscr{P}(y, T y, t), \frac{\mathscr{P}(x, T x, t) \mathscr{P}(y, T y, t)}{\mathscr{P}(x, y, t)}\right\} .
\end{aligned}
$$

(iii) Let $x, y \in[2, \infty)$. Then,

$$
\begin{aligned}
& \mathscr{P}(T x, T y, t) \\
& =t \max \left\{\frac{2}{5}, \frac{2}{5}\right\} \leq t \max \left\{\frac{6}{5}, \frac{6}{5}\right\} \\
& \leq t \max \left\{\frac{3}{5} x, \frac{3}{5} y\right\} \leq \frac{3}{5} t \max \{x, y\} \\
& \leq \frac{3}{5} \max \{\mathscr{P}(x, y, t), \mathscr{P}(x, T x, t), \\
& \left.\mathscr{P}(y, T y, t), \frac{\mathscr{P}(x, T x, t) \mathscr{P}(y, T y, t)}{\mathscr{P}(x, y, t)}\right\} .
\end{aligned}
$$

(iv) Let $x \in[0,1)$ and $y \in[1,2)$. Then,

$$
\begin{aligned}
& \mathscr{P}(T x, T y, t) \\
&=t \max \left\{\frac{1}{5} x^{2}, \frac{1}{5} y\right\} \leq \frac{3}{5} t \max \{x, y\} \\
& \leq \frac{3}{5} \max \\
&\{\mathscr{P}(x, y, t), \mathscr{P}(x, T x, t), \\
&\left.\mathscr{P}(y, T y, t), \frac{\mathscr{P}(x, T x, t) \mathscr{P}(y, T y, t)}{\mathscr{P}(x, y, t)}\right\} .
\end{aligned}
$$

(v) Let $x \in[0,1)$ and $y \in[2, \infty)$. Then,

$\mathscr{P}(T x, T y, t)$

$$
\begin{aligned}
&=t \max \left\{\frac{1}{5} x^{2}, \frac{2}{5}\right\} \leq t \max \left\{\frac{1}{5} x^{2}, \frac{6}{5}\right\} \\
& \leq t \max \left\{\frac{1}{5} x^{2}, \frac{3}{5} y\right\} \leq \frac{3}{5} t \max \{x, y\} \\
& \leq \frac{3}{5} \max \{\mathscr{P}(x, y, t), \mathscr{P}(x, T x, t), \\
&\left.\mathscr{P}(y, T y, t), \frac{\mathscr{P}(x, T x, t) \mathscr{P}(y, T y, t)}{\mathscr{P}(x, y, t)}\right\} .
\end{aligned}
$$

(vi) Let $x \in[1,2)$ and $y \in[2, \infty)$. Then,

$$
\begin{aligned}
\mathscr{P} & (T x, T y, t) \\
& =t \max \left\{\frac{1}{5} x, \frac{2}{5}\right\} \leq t \max \left\{\frac{1}{5} x, \frac{6}{5}\right\}
\end{aligned}
$$

$$
\begin{aligned}
& \leq t \max \left\{\frac{1}{5} x, \frac{3}{5} y\right\} \leq \frac{3}{5} t \max \{x, y\} \\
& \leq \frac{3}{5} \max \{\mathscr{P}(x, y, t), \mathscr{P}(x, T x, t) \\
& \left.\mathscr{P}(y, T y, t), \frac{\mathscr{P}(x, T x, t) \mathscr{P}(y, T y, t)}{\mathscr{P}(x, y, t)}\right\} .
\end{aligned}
$$

Therefore,

$$
\begin{aligned}
& \mathscr{P}(T x, T y, t) \\
& \leq \frac{3}{5} \max \{\mathscr{P}(x, y, t), \mathscr{P}(x, T x, t), \\
&\left.\mathscr{P}(y, T y, t), \frac{\mathscr{P}(x, T x, t) \mathscr{P}(y, T y, t)}{\mathscr{P}(x, y, t)}\right\},
\end{aligned}
$$

for all $x, y \in X$ with $x \neq y$ and all $t>0$. Hence, all conditions of Theorem 12 hold and $T$ has a unique fixed point.

Corollary 14. Let $(X, \mathscr{P})$ be a complete parametric metric space and let $T: X \rightarrow X$ be a continuous self-mapping such that

$$
\mathscr{P}(T x, T y, t) \leq r \max \{\mathscr{P}(x, T x, t), \mathscr{P}(y, T y, t)\}
$$

holds for all $x, y \in X$ and all $t>0$ where $0 \leq r<1$. Then $T$ has a unique fixed point.

Corollary 15. Let $(X, \mathscr{P})$ be a complete parametric metric space and let $T: X \rightarrow X$ be a continuous self-mapping such that

$$
\mathscr{P}(T x, T y, t) \leq \alpha \mathscr{P}(x, y, t)+\frac{\beta \mathscr{P}(x, T x, t) \mathscr{P}(y, T y, t)}{\mathscr{P}(x, y, t)}
$$

holds for all $x, y \in X$ with $x \neq y$ and all $t>0$ where $\alpha, \beta \geq 0$, with $\alpha+\beta<1$. Then $T$ has a unique fixed point.

Proof. Since

$$
\begin{aligned}
& \mathscr{P}(T x, T y, t) \\
& \leq \leq \mathscr{P}(x, y, t)+\frac{\beta \mathscr{P}(x, T x, t) \mathscr{P}(y, T y, t)}{\mathscr{P}(x, y, t)} \\
& \leq(\alpha+\beta) \max \left\{\mathscr{P}(x, y, t), \frac{\mathscr{P}(x, T x, t) \mathscr{P}(y, T y, t)}{\mathscr{P}(x, y, t)}\right\} \\
& \leq(\alpha+\beta) \max \{\mathscr{P}(x, y, t), \mathscr{P}(x, T x, t), \mathscr{P}(y, T y, t), \\
&\left.\frac{\mathscr{P}(x, T x, t) \mathscr{P}(y, T y, t)}{\mathscr{P}(x, y, t)}\right\}
\end{aligned}
$$


holds for all $x, y \in X$ with $x \neq y$ and all $t>0$, all conditions of Theorem 12 hold and $T$ has a unique fixed point.

Theorem 16. Let $(X, \mathscr{P})$ be a complete parametric metric space and let $T$ be a self-mapping on $X$. Assume that

$$
\begin{aligned}
\mathscr{P} & (T x, T y, t) \\
& \leq\left(\frac{\mathscr{P}(x, T y, t)+\mathscr{P}(y, T x, t)}{\mathscr{P}(x, T y, t)+\mathscr{P}(y, T x, t)+\ell(t)}\right) \mathscr{P}(x, y, t)
\end{aligned}
$$

holds for all $x, y \in X$ and all $t>0$ where $\ell:(0, \infty) \rightarrow(0, \infty)$ is a function. Then $T$ has a fixed point.

Proof. Let $x_{0} \in X$. Define a sequence $\left\{x_{n}\right\} \subset X$ by $x_{n+1}=T x_{n}$ for all positive integers $n$. From (23) with $x=x_{n}$ and $y=x_{n-1}$ we get

$$
\begin{aligned}
\mathscr{P}( & \left.x_{n+1}, x_{n}, t\right) \\
= & \mathscr{P}\left(T x_{n}, T x_{n-1}, t\right) \\
\leq & \left(\frac{\mathscr{P}\left(x_{n}, T x_{n-1}, t\right)+\mathscr{P}\left(x_{n-1}, T x_{n}, t\right)}{\mathscr{P}\left(x_{n}, T x_{n-1}, t\right)+\mathscr{P}\left(x_{n-1}, T x_{n}, t\right)+\ell(t)}\right) \\
& \times \mathscr{P}\left(x_{n}, x_{n-1}, t\right) \\
= & \left(\frac{\mathscr{P}\left(x_{n-1}, x_{n+1}, t\right)}{\mathscr{P}\left(x_{n-1}, x_{n+1}, t\right)+\ell(t)}\right) \\
& \times \mathscr{P}\left(x_{n}, x_{n-1}, t\right) \\
\leq & \left(\frac{\mathscr{P}\left(x_{n-1}, x_{n}, t\right)+\mathscr{P}\left(x_{n}, x_{n+1}, t\right)}{\mathscr{P}\left(x_{n-1}, x_{n}, t\right)+\mathscr{P}\left(x_{n}, x_{n+1}, t\right)+\ell(t)}\right) \\
& \times \mathscr{P}\left(x_{n}, x_{n-1}, t\right),
\end{aligned}
$$

which implies $\left\{\mathscr{P}\left(x_{n}, x_{n+1}, t\right)\right\}$ is a nonincreasing sequence and so

$$
\begin{aligned}
& \frac{\mathscr{P}\left(x_{n-1}, x_{n}, t\right)+\mathscr{P}\left(x_{n}, x_{n+1}, t\right)}{\mathscr{P}\left(x_{n-1}, x_{n}, t\right)+\mathscr{P}\left(x_{n}, x_{n+1}, t\right)+\ell(t)} \\
& \leq \frac{\mathscr{P}\left(x_{n-2}, x_{n-1}, t\right)+\mathscr{P}\left(x_{n-1}, x_{n}, t\right)}{\mathscr{P}\left(x_{n-2}, x_{n-1}, t\right)+\mathscr{P}\left(x_{n-1}, x_{n}, t\right)+\ell(t)} \\
& \leq \cdots \leq \frac{\mathscr{P}\left(x_{0}, x_{1}, t\right)+\mathscr{P}\left(x_{1}, x_{2}, t\right)}{\mathscr{P}\left(x_{0}, x_{1}, t\right)+\mathscr{P}\left(x_{0}, x_{1}, t\right)+\ell(t)} \\
& =r<1,
\end{aligned}
$$

for all $n \geq 2$. Therefore from (24) we get

$$
\begin{aligned}
& \mathscr{P}\left(x_{n+1}, x_{n}, t\right) \\
& \leq\left(\frac{\mathscr{P}\left(x_{n-1}, x_{n}, t\right)+\mathscr{P}\left(x_{n}, x_{n+1}, t\right)}{\mathscr{P}\left(x_{n-1}, x_{n}, t\right)+\mathscr{P}\left(x_{n}, x_{n+1}, t\right)+\ell(t)}\right) \\
& \quad \times \mathscr{P}\left(x_{n}, x_{n-1}, t\right) \leq r \mathscr{P}\left(x_{n}, x_{n-1}\right),
\end{aligned}
$$

for all $n \geq 2$ where $0 \leq r<1$. Now, it is easy to show that $\left\{x_{n}\right\}$ is a Cauchy sequence. The completeness of $X$ ensures that the sequence $\left\{x_{n}\right\}$ converges to some $x^{*} \in X$. From (23) we obtain

$$
\begin{aligned}
\mathscr{P} & \left.x_{n+1}, T x^{*}, t\right) \\
= & \mathscr{P}\left(T x_{n}, T x^{*}, t\right) \\
\leq & \left(\frac{\mathscr{P}\left(x_{n}, T x^{*}, t\right)+\mathscr{P}\left(x^{*}, T x_{n}, t\right)}{\mathscr{P}\left(x_{n}, T x^{*}, t\right)+\mathscr{P}\left(x^{*}, T x_{n}, t\right)+\ell(t)}\right) \\
& \times \mathscr{P}\left(x_{n}, x^{*}, t\right) .
\end{aligned}
$$

Taking limit as $n \rightarrow \infty$ in the above inequality, we deduce that $\mathscr{P}\left(x^{*}, T x^{*}, t\right)=0$; that is, $x^{*}=T x^{*}$.

Example 17. Let $X$ denote the set of all functions $f$ : $(0, \infty) \rightarrow[0, \infty)$. Define $\ell:(0, \infty) \rightarrow(0, \infty)$ by $\ell(t)=1$ and $\mathscr{P}: X^{2} \times X^{2} \times(0, \infty) \rightarrow[0, \infty)$ by

$$
\mathscr{P}((f, g),(h, j), t)=q(f(t), h(t))+q(g(t), j(t)),
$$

for all $f, g, h, j \in X$ and all $t>0$ where

$$
q(f(t), h(t))= \begin{cases}\max \{f(t), h(t)\}, & f \neq h \\ 0, & f=h .\end{cases}
$$

Then $\left(X^{2}, \mathscr{P}\right)$ is a complete parametric metric space. Define $T: X^{2} \rightarrow X^{2}$ by $T(f, g)=(1,(1 / 4) g)$. If $(f, g) \neq(1,(1 / 4) j)$ or $(h, j) \neq(1,(1 / 4) g)$, then

$$
\mathscr{P}((f, g), T(h, j))+\mathscr{P}((h, j), T(f, g)) \geq 1=\ell(t),
$$

and so,

$$
\begin{aligned}
2[ & \mathscr{P}((f, g), T(h, j), t)+\mathscr{P}((h, j), T(f, g), t)] \\
& \geq \mathscr{P}((f, g), T(h, j), t)+\mathscr{P}((h, j), T(f, g), t)+\ell(t),
\end{aligned}
$$

which implies

$$
\frac{1}{2} \leq \frac{\mathscr{P}((f, g), T(h, j), t)+\mathscr{P}((h, j), T(f, g), t)}{\mathscr{P}((f, g), T(h, j), t)+\mathscr{P}((h, j), T(f, g), t)+\ell(t)} .
$$

Now we get

$$
\begin{aligned}
\mathscr{P} & (T(h, j), T(f, g), t) \\
= & \mathscr{P}\left(\left(1, \frac{1}{4} j\right),\left(1, \frac{1}{4} g, t\right)\right) \\
= & \frac{1}{4} \max \{j(t), g(t)\} \\
\leq & \frac{1}{2}(\max \{h(t), f(t)\}+\max \{j(t), g(t)\}) \\
= & \frac{1}{2} \mathscr{P}((h, j),(f, g), t) \\
\leq & \frac{\mathscr{P}((f, g), T(h, j), t)+\mathscr{P}((h, j), T(f, g), t)}{\mathscr{P}((f, g), T(h, j), t)+\mathscr{P}((h, j), T(f, g), t)+\ell(t)} \\
& \times \mathscr{P}((h, j),(f, g), t) .
\end{aligned}
$$


Also if $(f, g)=(1,(1 / 4) j)$ and $(h, j)=(1,(1 / 4) g)$, then $f=$ $h=1, g=(1 / 4) j$, and $j=(1 / 4) g$. That is, $f=h=1$ and $g=j=0$, and so

$$
\begin{aligned}
\mathscr{P} & (T(h, j), T(f, g)) \\
= & \mathscr{P}\left(\left(1, \frac{1}{4} j\right),\left(1, \frac{1}{4} g\right)\right) \\
= & \mathscr{P}((1,0),(1,0)) \\
= & 0 \leq\left(\frac{\mathscr{P}((h, j), T(f, g), t)+\mathscr{P}((f, g), T(h, j), t)}{\mathscr{P}((h, j), T(f, g), t)+\mathscr{P}((f, g), T(h, j), t)+\ell(t)}\right) \\
& \times \mathscr{P}((h, j),(f, g), t) .
\end{aligned}
$$

Therefore, all conditions of Theorem 16 hold true and $T$ has a fixed point.

If we take $\mathscr{P}(x, y, t)=1 / M(x, y, t)-1$ in Corollaries 14 and 15 , respectively, we deduce the following recent results as corollaries.

Corollary 18 (Theorem 2.1 in [19]). Let $(X, M, N, *, \diamond)$ be a complete triangular intuitionistic fuzzy metric space and let $T$ : $X \rightarrow X$ be a continuous mapping satisfying the contractive condition

$$
\begin{aligned}
& \frac{1}{M(T x, T y, t)}-1 \\
& \quad \leq r \max \left\{\frac{1}{M(x, T x, t)}-1, \frac{1}{M(y, T y, t)}-1\right\},
\end{aligned}
$$

for all $x, y \in X$ and all $t>0$ where $0 \leq r<1$. Then $T$ has $a$ fixed point.

Corollary 19 (Theorem 2.3 in [19]). Let $(X, M, N, *, \diamond)$ be a complete triangular intuitionistic fuzzy metric space and let $T$ : $X \rightarrow X$ be a continuous mapping satisfying the contractive condition

$$
\begin{aligned}
& \frac{1}{M(T x, T y, t)}-1 \\
& \leq \alpha \frac{(1 / M(x, T x, t)-1)(1 / M(y, T y, t)-1)}{1 / M(x, y, t)-1} \\
& \quad+\beta\left(\frac{1}{M(x, y, t)}-1\right),
\end{aligned}
$$

for all $x, y \in X$ and all $t>0$ where $\alpha, \beta \in[0,1]$ and $0 \leq$ $\alpha+\beta<1$. Then $T$ has a unique fixed point.

By taking $\mathscr{P}(x, y, t)=1 / M(x, y, t)-1$ and $\ell(t)=1 / t$ in Theorem 16, we deduce the following result.

Corollary 20 (Theorem 2.2 in [19]). Let $(X, M, N, *, \diamond)$ be a complete triangular intuitionistic fuzzy metric space and let
$T: X \rightarrow X$ be a continuous mapping satisfying the contractive condition

$$
\begin{aligned}
& \frac{1}{M(T x, T y, t)}-1 \\
& \leq\left(\frac{1 / M(x, T y, t)-1+1 / M(y, T x, t)-1}{1 / M(x, T y, t)-1+1 / M(y, T x, t)-1+1 / t}\right) \\
& \quad \times\left(\frac{1}{M(x, y, t)}-1\right),
\end{aligned}
$$

for all $x, y \in X$ and all $t>0$. Then $T$ has a fixed point.

\section{A New Fixed Point Theorem in Intuitionistic Fuzzy Metric Spaces}

In this section we suggest new contraction and prove fixed point theorems in the framework of triangular intuitionistic fuzzy metric spaces which can not be obtained from the existing results in metric spaces.

Let $\Psi_{\mathscr{L}}$ denotes the class of those functions $\mathscr{L}:[0, \infty) \rightarrow$ $(0,1)$ which satisfies the condition $\mathscr{L}\left(t_{n}\right) \rightarrow 1 \Rightarrow t_{n} \rightarrow 1$.

Theorem 21. Let $(X, M, N, *, \diamond)$ be a complete triangular intuitionistic fuzzy metric space and let $T$ be a self-mapping on $X$ such that

$$
\begin{aligned}
\frac{1}{M(T x, T y, t)} \leq & \mathscr{L}\left(P^{T}(x, y, t)\right) P^{T}(x, y, t) \\
& +\left|Q^{T}(x, y, t)-\mathscr{L}\left(P^{T}(x, y, t)\right)\right|
\end{aligned}
$$

holds for all $x, y \in X$ and all $t>0$ where $\mathscr{L} \in \Psi_{\mathscr{L}}$,

$$
\begin{aligned}
& P^{T}(x, y, t) \\
& =\max \left\{\frac{1}{M(x, y, t)}, \frac{1}{M(x, T x, t)}, \frac{1}{M(y, T y, t)},\right. \\
& \left.\quad \frac{1}{2}\left[\frac{1}{M(x, T y, t)}+\frac{1}{M(y, T x, t)}-1\right]\right\}, \\
& Q^{T}(x, y, t) \\
& =\max \{M(x, y, t), M(x, T x, t), M(y, T y, t), \\
& M(x, T y, t), M(y, T x, t)\} .
\end{aligned}
$$

Then $T$ has a unique fixed point.

Proof. Let $x_{0} \in X$. We define an iterative sequence $\left\{x_{n}\right\}$ in the following way:

$$
x_{n}=T^{n} x_{0}=T x_{n-1} \quad \forall n \in \mathbb{N} \text {. }
$$


From (38) with $x=x_{n-1}$ and $y=x_{n}$ we get

$$
\begin{aligned}
& \frac{1}{M\left(x_{n}, x_{n+1}, t\right)} \\
& =\frac{1}{M\left(T x_{n-1}, T x_{n}, t\right)} \\
& \leq \mathscr{L}\left(P^{T}\left(x_{n-1}, x_{n}, t\right)\right) P^{T}\left(x_{n-1}, x_{n}, t\right) \\
& \quad+\left|Q^{T}\left(x_{n-1}, x_{n}, t\right)-\mathscr{L}\left(P^{T}\left(x_{n-1}, x_{n}, t\right)\right)\right|,
\end{aligned}
$$

for all $t>0$ and all $n \in \mathbb{N}$ where

$$
\begin{aligned}
& Q^{T}\left(x_{n-1}, x_{n}, t\right) \\
& =\max \left\{M\left(x_{n-1}, x_{n}, t\right), M\left(x_{n-1}, T x_{n-1}, t\right),\right. \\
& M\left(x_{n}, T x_{n}, t\right), M\left(x_{n-1}, T x_{n}, t\right), \\
& \left.M\left(x_{n}, T x_{n-1}, t\right)\right\} \\
& =\max \left\{M\left(x_{n-1}, x_{n}, t\right), M\left(x_{n}, x_{n+1}, t\right),\right. \\
& \left.M\left(x_{n-1}, x_{n+1}, t\right), M\left(x_{n}, x_{n}, t\right)\right\}=1 .
\end{aligned}
$$

Now from (41) and (42) we have

$$
\begin{aligned}
& \frac{1}{M\left(x_{n}, x_{n+1}, t\right)} \\
& \leq \mathscr{L}\left(P^{T}\left(x_{n-1}, x_{n}, t\right)\right) P^{T}\left(x_{n-1}, x_{n}, t\right) \\
&+\left|1-\mathscr{L}\left(P^{T}\left(x_{n-1}, x_{n}, t\right)\right)\right| \\
&= \mathscr{L}\left(P^{T}\left(x_{n-1}, x_{n}, t\right)\right) P^{T}\left(x_{n-1}, x_{n}, t\right) \\
&+1-\mathscr{L}\left(P^{T}\left(x_{n-1}, x_{n}, t\right)\right) \\
&= \mathscr{L}\left(P^{T}\left(x_{n-1}, x_{n}, t\right)\right)\left[P^{T}\left(x_{n-1}, x_{n}, t\right)-1\right]+1,
\end{aligned}
$$

which implies

$$
\begin{aligned}
& \frac{1}{M\left(x_{n}, x_{n+1}, t\right)}-1 \\
& \quad \leq \mathscr{L}\left(P^{T}\left(x_{n-1}, x_{n}, t\right)\right)\left[P^{T}\left(x_{n-1}, x_{n}, t\right)-1\right],
\end{aligned}
$$

where

$$
\begin{aligned}
P^{T}\left(x_{n-1}, x_{n}, t\right) & \\
=\max & \left\{\frac{1}{M\left(x_{n-1}, x_{n}, t\right)},\right. \\
& \frac{1}{M\left(x_{n-1}, T x_{n-1}, t\right)}, \frac{1}{M\left(x_{n}, T x_{n}, t\right)},
\end{aligned}
$$$$
\frac{1}{2}\left[\frac{1}{M\left(x_{n-1}, T x_{n}, t\right)}\right.
$$$$
\left.\left.+\frac{1}{M\left(x_{n}, T x_{n-1}, t\right)}-1\right]\right\}
$$$$
=\max \left\{\frac{1}{M\left(x_{n-1}, x_{n}, t\right)},\right.
$$

$$
\left.\frac{1}{M\left(x_{n}, x_{n+1}, t\right)}, \frac{1}{2 M\left(x_{n-1}, x_{n+1}, t\right)}\right\}
$$$$
\leq \max \left\{\frac{1}{M\left(x_{n-1}, x_{n}, t\right)}, \frac{1}{M\left(x_{n}, x_{n+1}, t\right)},\right.
$$$$
\frac{1}{2}\left[\frac{1}{M\left(x_{n-1}, x_{n}, t\right)}-1\right.
$$$$
\left.\left.+\frac{1}{M\left(x_{n}, x_{n+1}, t\right)}-1\right]+\frac{1}{2}\right\}
$$$$
\leq \max \left\{\frac{1}{M\left(x_{n-1}, x_{n}, t\right)}, \frac{1}{M\left(x_{n}, x_{n+1}, t\right)},\right.
$$$$
\left.\frac{1}{2}\left[\frac{1}{M\left(x_{n-1}, x_{n}, t\right)}+\frac{1}{M\left(x_{n}, x_{n+1}, t\right)}\right]-\frac{1}{2}\right\}
$$$$
=\max \left\{\frac{1}{M\left(x_{n-1}, x_{n}, t\right)}, \frac{1}{M\left(x_{n}, x_{n+1}, t\right)}\right\} \text {. }
$$

Thus

$$
P^{T}\left(x_{n-1}, x_{n}, t\right)=\max \left\{\frac{1}{M\left(x_{n-1}, x_{n}, t\right)}, \frac{1}{M\left(x_{n}, x_{n+1}, t\right)}\right\} .
$$

Now if $P^{T}\left(x_{n-1}, x_{n}, t\right)=1 / M\left(x_{n}, x_{n+1}, t\right)$, then by (44) we obtain

$$
\begin{aligned}
& \frac{1}{M\left(x_{n}, x_{n+1}, t\right)}-1 \\
& \quad \leq \mathscr{L}\left(\frac{1}{M\left(x_{n}, x_{n+1}, t\right)}\right)\left[\frac{1}{M\left(x_{n}, x_{n+1}, t\right)}-1\right] \\
& \quad<\frac{1}{M\left(x_{n}, x_{n+1}, t\right)}-1,
\end{aligned}
$$

which is a contradiction. Hence,

$$
\begin{aligned}
& \frac{1}{M\left(x_{n}, x_{n+1}, t\right)}-1 \\
& \quad \leq \mathscr{L}\left(\frac{1}{M\left(x_{n-1}, x_{n}, t\right)}\right)\left[\frac{1}{M\left(x_{n-1}, x_{n}, t\right)}-1\right] \\
& \quad<\frac{1}{M\left(x_{n-1}, x_{n}, t\right)}-1,
\end{aligned}
$$


for all $n \in \mathbb{N}$ and all $t>0$. Therefore $\left\{1 / M\left(x_{n}, x_{n+1}, t\right)-1\right\}$ is a nonincreasing sequence and so it converges to some $s \geq 0$. Suppose to the contrary that $s>0$. Taking limit as $n \rightarrow \infty$ in $(48)$ we get $\lim _{n \rightarrow \infty} \mathscr{L}\left(1 / M\left(x_{n-1}, x_{n}, t\right)\right)=1$. Due to property of $\mathscr{L}$ we have $\lim _{n \rightarrow \infty}\left(1 / M\left(x_{n-1}, x_{n}, t\right)\right)=1$. Equivalently, $\lim _{n \rightarrow \infty}\left[1 / M\left(x_{n-1}, x_{n}, t\right)-1\right]=0$, which is a contradiction. Thus,

$$
\lim _{n \rightarrow \infty} M\left(x_{n-1}, x_{n}, t\right)=1 \text {. }
$$

Now we want to show that $\lim _{\sup } \sin _{m \rightarrow \infty} M\left(x_{n}, x_{m}, t\right)=1$ for all $t>0$. Suppose to the contrary

$$
\lambda:=\limsup _{m, n \rightarrow \infty} M\left(x_{n}, x_{m}, t\right)<1 .
$$

From (38) we have

$$
\begin{aligned}
\frac{1}{M\left(x_{n+1}, x_{m+1}, t\right)} \\
=\frac{1}{M\left(T x_{n}, T x_{m}, t\right)} \\
\leq \mathscr{L}\left(P^{T}\left(x_{n}, x_{m}, t\right)\right) P^{T}\left(x_{n}, x_{m}, t\right) \\
\quad+\left|Q^{T}\left(x_{n}, x_{m}, t\right)-\mathscr{L}\left(P^{T}\left(x_{n}, x_{m}, t\right)\right)\right| .
\end{aligned}
$$

On the other hand,

$$
\begin{aligned}
P^{T} & \left(x_{n}, x_{m}, t\right) \\
& =\max \left\{\frac{1}{M\left(x_{n}, x_{m}, t\right)}, \frac{1}{M\left(x_{n}, T x_{n}, t\right)}, \frac{1}{M\left(x_{m}, T x_{m}, t\right)},\right. \\
& \left.=\max \left\{\frac{1}{M\left(x_{n}, x_{m}, t\right)}, \frac{1}{M\left(x_{n}, x_{n+1}, t\right)}, \frac{1}{M\left(x_{m}, x_{m+1}, t\right)}, \frac{1}{M\left(x_{m}, T x_{n}, t\right)}-1\right]\right\} \\
& \left.=\frac{1}{M}\left[\frac{1}{M\left(x_{n}, x_{m+1}, t\right)}+\frac{1}{M\left(x_{m}, x_{n+1}, t\right)}-1\right]\right\}, \\
& \left(x_{n}, x_{m+1}, t\right) \\
& \leq \frac{1}{M\left(x_{n}, x_{m+1}, t\right)}-1+1 \\
& +\frac{1}{M\left(x_{n}, x_{m}, t\right)}-1 \\
& +\frac{1}{M\left(x_{n}, x_{m}, t\right)}+\frac{1}{M\left(x_{m}, x_{m+1}, t\right)}-1 . \\
& \frac{1}{M}+1
\end{aligned}
$$

Similarly,

$$
\frac{1}{M\left(x_{m}, x_{n+1}, t\right)} \leq \frac{1}{M\left(x_{m}, x_{n}, t\right)}+\frac{1}{M\left(x_{n}, x_{n+1}, t\right)}-1,
$$

so from (52) and (53) we obtain

$$
\begin{aligned}
& P^{T}\left(x_{n}, x_{m}, t\right) \max \left\{\frac{1}{M\left(x_{n}, x_{m}, t\right)}, \frac{1}{M\left(x_{n}, x_{n+1}, t\right)}, \frac{1}{M\left(x_{m}, x_{m+1}, t\right)},\right. \\
&\left.\frac{1}{2}\left[\frac{1}{M\left(x_{n}, x_{m+1}, t\right)}+\frac{1}{M\left(x_{m}, x_{n+1}, t\right)}-1\right]\right\} \\
& \leq \max \left\{\frac{1}{M\left(x_{n}, x_{m}, t\right)}, \frac{1}{M\left(x_{n}, x_{n+1}, t\right)}, \frac{1}{M\left(x_{m}, x_{m+1}, t\right)},\right. \\
& \frac{1}{2}\left[\frac{1}{M\left(x_{n}, x_{m}, t\right)}+\frac{1}{M\left(x_{m}, x_{m+1}, t\right)}-1\right. \\
&\left.\left.\quad+\frac{1}{M\left(x_{m}, x_{n}, t\right)}+\frac{1}{M\left(x_{n}, x_{n+1}, t\right)}-1-1\right]\right\} .
\end{aligned}
$$

Taking limit supremum as $m, n \rightarrow \infty$ in (54) and using (49) and (50) we deduce

$$
\begin{aligned}
\frac{1}{\lambda} & =\limsup _{m, n \rightarrow \infty} \frac{1}{M\left(x_{n}, x_{m}, t\right)} \\
& \leq \lim _{m, n \rightarrow \infty} \sup ^{T}\left(x_{n}, x_{m}, t\right) \\
& \leq \max \left\{\frac{1}{\lambda}, 1,1,\right. \\
& \left.=\frac{1}{2}\left[\frac{1}{\lambda}+1-1+\frac{1}{\lambda}+1-1-1\right]\right\} \\
& =\max \left\{\frac{1}{\lambda}, 1, \frac{1}{\lambda}-\frac{1}{2}\right\}=\frac{1}{\lambda},
\end{aligned}
$$

which implies

$$
\limsup _{m, n \rightarrow \infty} P^{T}\left(x_{n}, x_{m}, t\right)=\frac{1}{\lambda} .
$$

Further,

$$
\begin{aligned}
\limsup _{m, n \rightarrow \infty} Q^{T}\left(x_{n}, x_{m}, t\right) & \\
=\max & \left\{\limsup _{m, n \rightarrow \infty} M\left(x_{n}, x_{m}, t\right),\right. \\
& \limsup _{m, n \rightarrow \infty} M\left(x_{n}, T x_{n}, t\right),
\end{aligned}
$$




$$
\begin{aligned}
& \limsup _{m, n \rightarrow \infty} M\left(x_{m}, T x_{m}, t\right), \\
& \limsup _{m, n \rightarrow \infty} M\left(x_{n}, T x_{m}, t\right), \\
& \left.\limsup _{m, n \rightarrow \infty} M\left(x_{m}, T x_{n}, t\right)\right\} \\
=\max & \left\{\limsup _{m, n \rightarrow \infty} M\left(x_{n}, x_{m}, t\right),\right. \\
& \limsup _{m, n \rightarrow \infty} M\left(x_{n}, x_{n+1}, t\right), \\
& \limsup _{m, n \rightarrow \infty} M\left(x_{m}, x_{m+1}, t\right), \\
& \limsup _{m, n \rightarrow \infty} M\left(x_{n}, x_{m+1}, t\right), \\
& \left.\limsup _{m, n \rightarrow \infty} M\left(x_{m}, x_{n+1}, t\right)\right\} \\
=\max \{ & \left.\left\{\begin{array}{l}
\lambda, 1,1, \lim _{m, n \rightarrow \infty} \sup _{m} M\left(x_{n}, x_{m+1}, t\right), \\
m, n \rightarrow \infty
\end{array}, x_{m}, x_{n+1}, t\right)\right\}=1 .
\end{aligned}
$$

Taking limit supremum as $m, n \rightarrow \infty$ in (51) and applying (56) and (57) we get

$$
\begin{aligned}
\lim _{m, n \rightarrow \infty} \sup _{m\left(x_{n+1}, x_{m+1}, t\right)} & \frac{1}{\leq} \lim _{m, n \rightarrow \infty} \sup _{\mathscr{L}}\left(P^{T}\left(x_{n}, x_{m}, t\right)\right) \limsup _{m, n \rightarrow \infty} P^{T}\left(x_{n}, x_{m}, t\right) \\
& +\left|\limsup _{m, n \rightarrow \infty} Q^{T}\left(x_{n}, x_{m}, t\right)-\limsup _{m, n \rightarrow \infty} \mathscr{L}\left(P^{T}\left(x_{n}, x_{m}, t\right)\right)\right| \\
= & \frac{1}{\lambda} \limsup _{m, n \rightarrow \infty} \mathscr{L}\left(P^{T}\left(x_{n}, x_{m}, t\right)\right) \\
& +\left|1-\limsup _{m, n \rightarrow \infty} \mathscr{L}\left(P^{T}\left(x_{n}, x_{m}, t\right)\right)\right| \\
= & \left(\frac{1}{\lambda}-1\right) \limsup _{m, n \rightarrow \infty} \mathscr{L}\left(P^{T}\left(x_{n}, x_{m}, t\right)\right)+1 .
\end{aligned}
$$

On the other hand we have

$$
\begin{aligned}
& \frac{1}{M\left(x_{n}, x_{m}, t\right)}-1 \\
& \leq \frac{1}{M\left(x_{n}, x_{n+1}, t\right)}-1+\frac{1}{M\left(x_{n+1}, x_{m}, t\right)}-1 \\
& \leq \frac{1}{M\left(x_{n}, x_{n+1}, t\right)}-1+\frac{1}{M\left(x_{n+1}, x_{m+1}, t\right)}-1 \\
& \quad+\frac{1}{M\left(x_{m+1}, x_{m}, t\right)}-1
\end{aligned}
$$

By taking limit supremum as $m, n \rightarrow \infty$ in the above inequality and using (49) and (58) we get

$$
\begin{aligned}
\frac{1}{\lambda}-1 & \leq \limsup _{m, n \rightarrow \infty} \frac{1}{M\left(x_{n+1}, x_{m+1}, t\right)}-1 \\
& \leq\left(\frac{1}{\lambda}-1\right) \limsup _{m, n \rightarrow \infty} \mathscr{L}\left(P^{T}\left(x_{n}, x_{m}, t\right)\right)
\end{aligned}
$$

which implies $\lim \sup _{m, n \rightarrow \infty} \mathscr{L}\left(P^{T}\left(x_{n}, x_{m}, t\right)\right)=1$. Thus, $\lim _{\sup _{m, n \rightarrow \infty}} P^{T}\left(x_{n}, x_{m}, t\right)=1$ which is a contradiction. Hence, $\lim \sup _{m, n \rightarrow \infty} M\left(x_{n}, x_{m}, t\right)=1$. That is, $\left\{x_{n}\right\}$ is a Cauchy sequence. Since $X$ is complete intuitionistic fuzzy metric spaces, there exists $x^{*} \in X$ such that $x_{n} \rightarrow x^{*}$ as $n \rightarrow \infty$. From (38) we have

$$
\begin{aligned}
\frac{1}{M\left(x_{n+1}, T x^{*}, t\right)} \\
=\frac{1}{M\left(T x_{n}, T x^{*}, t\right)} \\
\leq \\
\quad \mathscr{L}\left(P^{T}\left(x_{n}, x^{*}, t\right)\right) P^{T}\left(x_{n}, x^{*}, t\right) \\
\quad+\left|Q^{T}\left(x_{n}, x^{*}, t\right)-\mathscr{L}\left(P^{T}\left(x_{n}, x^{*}, t\right)\right)\right|,
\end{aligned}
$$

for all $t>0$ where

$$
\begin{aligned}
P^{T}\left(x_{n}, x^{*}, t\right) & \\
=\max & \left\{\frac{1}{M\left(x_{n}, x^{*}, t\right)}, \frac{1}{M\left(x_{n}, T x_{n}, t\right)}, \frac{1}{M\left(x^{*}, T x^{*}, t\right)},\right. \\
& \left.\frac{1}{2}\left[\frac{1}{M\left(x_{n}, T x^{*}, t\right)}+\frac{1}{M\left(x^{*}, T x_{n}, t\right)}-1\right]\right\} \\
=\max & \left\{\frac{1}{M\left(x_{n}, x^{*}, t\right)}, \frac{1}{M\left(x_{n}, x_{n+1}, t\right)}, \frac{1}{M\left(x^{*}, T x^{*}, t\right)},\right. \\
& \left.\frac{1}{2}\left[\frac{1}{M\left(x_{n}, T x^{*}, t\right)}+\frac{1}{M\left(x^{*}, x_{n+1}, t\right)}-1\right]\right\},
\end{aligned}
$$

and so

$$
\lim _{n \rightarrow \infty} P^{T}\left(x_{n}, x^{*}, t\right)=\frac{1}{M\left(x^{*}, T x^{*}, t\right)} .
$$

Note that

$$
\begin{aligned}
& Q^{T}\left(x_{n}, x^{*}, t\right) \\
& =\max \left\{M\left(x_{n}, x^{*}, t\right), M\left(x_{n}, x_{n+1}, t\right),\right. \\
& M\left(x^{*}, T x^{*}, t\right), M\left(x_{n}, T x^{*}, t\right), \\
& \left.M\left(x^{*}, x_{n+1}, t\right)\right\},
\end{aligned}
$$

and so

$$
\lim _{n \rightarrow \infty} Q^{T}\left(x_{n}, x^{*}, t\right)=1 \text {. }
$$


By taking limit as $n \rightarrow \infty$ in (61) we get

$$
\begin{aligned}
& \frac{1}{M\left(x^{*}, T x^{*}, t\right)}-1 \\
& \quad \leq \lim _{n \rightarrow \infty} \mathscr{L}\left(P^{T}\left(x_{n}, x^{*}, t\right)\right)\left(\frac{1}{M\left(x^{*}, T x^{*}, t\right)}-1\right) .
\end{aligned}
$$

Now, if $M\left(x^{*}, T x^{*}, t\right)<1$, then $\lim _{n \rightarrow \infty} \mathscr{L}\left(P^{T}\left(x_{n}, x^{*}, t\right)\right)=$ 1. Hence,

$$
\lim _{n \rightarrow \infty} P^{T}\left(x_{n}, x^{*}, t\right)=\frac{1}{M\left(x^{*}, T x^{*}, t\right)}=1,
$$

which is a contradiction. Thus, for all $t>0 M\left(x^{*}, T x^{*}, t\right)=$ 1 ; that is, $x^{*}=T x^{*}$. To prove the uniqueness, suppose that $u \neq v$, such that $T v=v$ and $T u=u$. From (38) we get

$$
\begin{aligned}
& \frac{1}{M(u, v, t)} \\
& =\frac{1}{M(T u, T v, t)} \leq \mathscr{L}\left(P^{T}(u, v, t)\right) P^{T}(u, v, t) \\
& \quad+\left|Q^{T}(u, v, t)-\mathscr{L}\left(P^{T}(u, v, t)\right)\right|,
\end{aligned}
$$

where

$$
\begin{aligned}
& P^{T}(u, v, t) \\
& =\max \left\{\frac{1}{M(u, v, t)}, \frac{1}{M(u, T u, t)}, \frac{1}{M(v, T v, t)},\right. \\
& \left.\quad \frac{1}{2}\left[\frac{1}{M(u, T v, t)}+\frac{1}{M(v, T u, t)}-1\right]\right\} \\
& =\max \left\{\frac{1}{M(u, v, t)}, 1,1, \frac{1}{M(u, v, t)}-\frac{1}{2}\right\} \\
& \left.Q^{T}=\frac{1}{M(u, v, t)}, v, t\right) \\
& =\max \{M(u, v, t), M(u, T u, t), \\
& \quad M(v, T v, t), M(u, T v, t), M(v, T u, t)\}=1 .
\end{aligned}
$$

Hence,

$$
\begin{aligned}
& \frac{1}{M(u, v, t)} \\
& \quad \leq \mathscr{L}\left(\frac{1}{M(u, v, t)}\right) \frac{1}{M(u, v, t)}+\left|1-\mathscr{L}\left(\frac{1}{M(u, v, t)}\right)\right| \\
& \quad=\mathscr{L}\left(P^{T}(u, v, t)\right)\left(\frac{1}{M(u, v, t)}-1\right)+1,
\end{aligned}
$$

which implies,

$$
\begin{aligned}
& \frac{1}{M(u, v, t)}-1 \\
& \quad \leq \mathscr{L}\left(P^{T}(u, v, t)\right)\left(\frac{1}{M(u, v, t)}-1\right)<\frac{1}{M(u, v, t)}-1
\end{aligned}
$$

which is a contradiction. So, $u=v$.

If in Theorem 21 we take $\mathscr{L}(t)=\lambda$ where $0 \leq \lambda<1$, then we deduce the following Corollary.

Corollary 22. Let $(X, M, N, *, \diamond)$ be a complete triangular intuitionistic fuzzy metric space and let $T$ be a self-mapping on $X$ such that

$$
\frac{1}{M(T x, T y, t)} \leq \lambda P^{T}(x, y, t)+\left|Q^{T}(x, y, t)-\lambda\right|
$$

holds for all $x, y \in X$ and all $t>0$ where $0 \leq \lambda<1$. Then $T$ has a unique fixed point.

Example 23. Let $X=\{0,1,3,4\}$ be a set with usual metric. Define intuitionistic fuzzy metric by

$$
\begin{aligned}
& M(x, y, t)=\frac{1}{1+|x-y|}, \\
& N(x, y, t)=\frac{|x-y|}{1+|x-y|},
\end{aligned}
$$

where $a * b=\min \{a, b\}$ and $a \diamond b=\max \{a, b\}$. Then $X$ is a complete triangular intuitionistic fuzzy metric space. Also define $T: X \rightarrow X$ by

$$
T x= \begin{cases}0, & x \in\{0,1\} \\ 1, & x \in\{3,4\} .\end{cases}
$$

Now we consider the following cases.

(i) Let $x=0$ and $y=1$. Then,

$$
\begin{aligned}
& P^{T}(0,1, t) \\
& =\max \left\{\frac{1}{M(0,1, t)}, \frac{1}{M(0, T 0, t)}, \frac{1}{M(1, T 1, t)},\right. \\
& \left.\quad \frac{1}{2}\left[\frac{1}{M(0, T 1, t)}+\frac{1}{M(1, T 0, t)}-1\right]\right\} \\
& =\max \left\{\frac{1}{M(0,1, t)}, \frac{1}{M(0,0, t)}, \frac{1}{M(1,0, t)},\right. \\
& \left.\quad \frac{1}{2}\left[\frac{1}{M(0,0, t)}+\frac{1}{M(1,0, t)}-1\right]\right\}=2, \\
& Q^{T}(0,1, t) \quad \max \{M(0,1, t), M(0, T 0, t), M(1, T 1, t), \\
& M(0, T 1, t), M(1, T 0, t)\}=1 .
\end{aligned}
$$


So,

$$
\begin{aligned}
& \frac{1}{M(T 0, T 1, t)} \\
& =1 \leq \frac{3}{4} \cdot 2+\left|1-\frac{3}{4}\right| \\
& =\frac{3}{4} P^{T}(0,1, t)+\left|Q^{T}(0,1, t)-\frac{3}{4}\right| \text {. }
\end{aligned}
$$

(ii) Let $x=3$ and $y=4$. Then,

$$
\begin{aligned}
& P^{T}(3,4, t) \\
& =\max \left\{\frac{1}{M(3,4, t)}, \frac{1}{M(3, T 3, t)}, \frac{1}{M(4, T 4, t)},\right. \\
& \left.\frac{1}{2}\left[\frac{1}{M(3, T 4, t)}+\frac{1}{M(4, T 3, t)}-1\right]\right\} \\
& =\max \left\{\frac{1}{M(3,4, t)}, \frac{1}{M(3,1, t)}, \frac{1}{M(4,1, t)},\right. \\
& \left.\quad \frac{1}{2}\left[\frac{1}{M(3,1, t)}+\frac{1}{M(4,1, t)}-1\right]\right\}=4, \\
& Q^{T}(3,4, t) \quad \max \{M(3,4, t), M(3, T 3, t), M(4, T 4, t), \\
& \quad M(3, T 4, t), M(4, T 3, t)\} \\
& =\max \{M(3,4, t), M(3,1, t), M(4,1, t), \\
& M(3,1, t), M(4,1, t)\}=\frac{1}{2} .
\end{aligned}
$$

Thus

$$
\begin{aligned}
& \frac{1}{M}(T 3, T 4, t) \\
& \quad=1 \leq \frac{3}{4} \cdot 4+\left|\frac{1}{2}-\frac{3}{4}\right| \\
& \quad=\frac{3}{4} P^{T}(3,4, t)+\left|Q^{T}(3,4, t)-\frac{3}{4}\right| .
\end{aligned}
$$

(iii) Let $x=0$ and $y=3$. Then,

$$
\begin{aligned}
P^{T}(0,3, t) & \\
=\max & \left\{\frac{1}{M(0,3, t)}, \frac{1}{M(0, T 0, t)}, \frac{1}{M(3, T 3, t)},\right. \\
& \left.\frac{1}{2}\left[\frac{1}{M(0, T 3, t)}+\frac{1}{M(3, T 0, t)}-1\right]\right\}
\end{aligned}
$$

$$
\begin{gathered}
=\max \left\{\frac{1}{M(0,3, t)}, \frac{1}{M(0,0, t)}, \frac{1}{M(3,1, t)},\right. \\
\left.\frac{1}{2}\left[\frac{1}{M(0,1, t)}+\frac{1}{M(3,0, t)}-1\right]\right\}=4, \\
Q^{T}(0,3, t) \\
=\max \{M(0,3, t), M(0, T 0, t), M(3, T 3, t), \\
\quad M(0, T 3, t), M(3, T 0, t)\} \\
=\max \{M(0,3, t), M(0,0, t), M(3, T 3, t), \\
M(0,1, t), M(3,0, t)\}=1 .
\end{gathered}
$$

Thus

$$
\begin{aligned}
\frac{1}{M(T 0, T 3, t)} & =2 \leq \frac{3}{4} \cdot 4+\left|1-\frac{3}{4}\right| \\
& =\frac{3}{4} P^{T}(0,3, t)+\left|Q^{T}(0,3, t)-\frac{3}{4}\right|
\end{aligned}
$$

(iv) Let $x=0$ and $y=4$. Then

$$
\begin{aligned}
& P^{T}(0,4, t) \\
& =\max \left\{\frac{1}{M(0,4, t)}, \frac{1}{M(0, T 0, t)}, \frac{1}{M(4, T 4, t)},\right. \\
& \left.\quad \frac{1}{2}\left[\frac{1}{M(0, T 4, t)}+\frac{1}{M(4, T 0, t)}-1\right]\right\} \\
& =\max \left\{\frac{1}{M(0,4, t)}, \frac{1}{M(0,0, t)}, \frac{1}{M(4,1, t)},\right. \\
& \left.\quad \frac{1}{2}\left[\frac{1}{M(0,1, t)}+\frac{1}{M(4,0, t)}-1\right]\right\}=5, \\
& Q^{T}(0,4, t) \quad \max \{M(0,4, t), M(0, T 0, t), M(4, T 4, t), \\
& \quad M(0, T 4, t), M(4, T 0, t)\} \\
& =\max \{M(0,4, t), M(0,0, t), M(4, T 4, t), \\
& \quad M(0,1, t), M(4,0, t)\}=1 .
\end{aligned}
$$

Thus,

$$
\begin{aligned}
\frac{1}{M(T 0, T 4, t)} & =2 \leq \frac{3}{4} \cdot 5+\left|1-\frac{3}{4}\right| \\
& =\frac{3}{4} P^{T}(0,4, t)+\left|Q^{T}(0,4, t)-\frac{3}{4}\right| .
\end{aligned}
$$


(v) Let $x=1$ and $y=3$. Then,

$P^{T}(1,3, t)$

$$
\begin{aligned}
=\max & \left\{\frac{1}{M(1,3, t)}, \frac{1}{M(1, T 1, t)}, \frac{1}{M(3, T 3, t)},\right. \\
& \left.\frac{1}{2}\left[\frac{1}{M(1, T 3, t)}+\frac{1}{M(3, T 1, t)}-1\right]\right\} \\
= & \max \left\{\frac{1}{M(1,3, t)}, \frac{1}{M(1,0, t)}, \frac{1}{M(3,1, t)},\right. \\
& \left.\frac{1}{2}\left[\frac{1}{M(1,1, t)}+\frac{1}{M(3,0, t)}-1\right]\right\}=3,
\end{aligned}
$$

$$
\begin{aligned}
& Q^{T}(1,3, t) \\
& =\max \{M(1,3, t), M(1, T 1, t), M(3, T 3, t), \\
& M(1, T 3, t), M(3, T 1, t)\} \\
& =\max \{M(1,3, t), M(1,0, t), M(3,1, t), \\
& M(1,1, t), M(3,0, t)\}=1 .
\end{aligned}
$$

Hence

$$
\begin{aligned}
\frac{1}{M(T 1, T 3, t)} & =2 \leq \frac{3}{4} \cdot 3+\left|1-\frac{3}{4}\right| \\
& =\frac{3}{4} P^{T}(1,3, t)+\left|Q^{T}(1,3, t)-\frac{3}{4}\right|
\end{aligned}
$$

(vi) Let $x=1$ and $y=4$. Then

$$
P^{T}(1,4, t)
$$

$$
\begin{aligned}
&=\max \left\{\frac{1}{M(1,4, t)}, \frac{1}{M(1, T 1, t)}, \frac{1}{M(4, T 4, t)},\right. \\
&\left.\frac{1}{2}\left[\frac{1}{M(1, T 4, t)}+\frac{1}{M(4, T 1, t)}-1\right]\right\} \\
&=\max \left\{\frac{1}{M(1,4, t)}, \frac{1}{M(1,0, t)}, \frac{1}{M(4,1, t)},\right. \\
&\left.\quad \frac{1}{2}\left[\frac{1}{M(1,1, t)}+\frac{1}{M(4,0, t)}-1\right]\right\}=4,
\end{aligned}
$$

$Q^{T}(1,4, t)$

$=\max \{M(1,4, t), M(1, T 1, t), M(4, T 4, t)$,

$$
M(1, T 4, t), M(4, T 1, t)\}
$$

$=\max \{M(1,4, t), M(1,0, t), M(4,1, t)$,

$$
M(1,1, t), M(4,0, t)\}=1 .
$$

So we obtain

$$
\begin{aligned}
\frac{1}{M(T 1, T 4, t)} & =2 \leq \frac{3}{4} \cdot 4+\left|1-\frac{3}{4}\right| \\
& =\frac{3}{4} P^{T}(1,4, t)+\left|Q^{T}(1,4, t)-\frac{3}{4}\right| .
\end{aligned}
$$

Therefore all conditions of Corollary 22 (Theorem 21) hold and $T$ has a unique fixed point.

\section{A Suzuki Type Fixed Point Theorem}

In 2008, Suzuki proved a remarkable fixed point theorem that is a generalization of the Banach contraction principle and characterizes the metric completeness. Consequently, a number of extensions and generalizations of this result appeared in the literature (see [20-29] and references therein). In this section we prove and suggest a Suzuki type fixed point theorem in the setting of intuitionistic fuzzy metric space that can not be obtained from the existing results in metric spaces.

Theorem 24. Let $(X, M, N, *, \diamond)$ be a complete triangular intuitionistic fuzzy metric space and let $T$ be a self-mapping on $X$. Assume that there exists $r \in(0,1)$ such that

$$
\begin{aligned}
& \frac{1}{1+r}\left(\frac{1}{M(x, T x, t)}-1\right) \\
& \quad \leq \frac{1}{M(x, y, t)}-1 \Longrightarrow \frac{1}{M(T x, T y, t)} \\
& \quad \leq \frac{r}{M(x, y, t)}+(1-r) M(y, T x, t),
\end{aligned}
$$

for all $x, y \in X$ and all $t>0$. Then $T$ has a unique fixed point. Proof. Let $x_{0} \in X$. We define an iterative sequence $\left\{x_{n}\right\}$ by

$$
x_{n}=T^{n} x_{0}=T x_{n-1} \quad \forall n \in \mathbb{N} .
$$

Now since

$$
\begin{aligned}
\frac{1}{1+r}\left(\frac{1}{M\left(x_{n-1}, T x_{n-1}, t\right)}-1\right) & \leq \frac{1}{M\left(x_{n-1}, T x_{n-1}, t\right)}-1 \\
& =\frac{1}{M\left(x_{n-1}, x_{n}, t\right)}-1
\end{aligned}
$$

holds for all $n \in \mathbb{N}$, from (88) we get

$$
\begin{aligned}
& \frac{1}{M\left(x_{n}, x_{n+1}, t\right)} \\
& =\frac{1}{M\left(T x_{n-1}, T x_{n}, t\right)} \leq \frac{r}{M\left(x_{n-1}, x_{n}, t\right)} \\
& \quad+(1-r) M\left(x_{n}, T x_{n-1}, t\right) \leq \frac{r}{M\left(x_{n-1}, x_{n}, t\right)} \\
& \quad+(1-r) M\left(x_{n}, x_{n}, t\right)=\frac{r}{M\left(x_{n-1}, x_{n}, t\right)}+1-r,
\end{aligned}
$$

which implies

$$
\frac{1}{M\left(x_{n}, x_{n+1}, t\right)}-1 \leq r\left(\frac{1}{M\left(x_{n-1}, x_{n}, t\right)}-1\right) .
$$


Hence

$$
\frac{1}{M\left(x_{n}, x_{n+1}, t\right)}-1 \leq r^{n}\left(\frac{1}{M\left(x_{0}, x_{1}, t\right)}-1\right) .
$$

By (92) and triangular property of intuitionistic fuzzy metric space we get

$$
\begin{aligned}
& \frac{1}{M\left(x_{n}, x_{m}, t\right)}-1 \\
& \leq \frac{1}{M\left(x_{n}, x_{n+1}, t\right)}-1+\frac{1}{M\left(x_{n+1}, x_{n+2}, t\right)}-1 \\
& \quad+\cdots+\frac{1}{M\left(x_{m-1}, x_{m}, t\right)}-1 \\
& \leq\left(r^{n}+r^{n+1}+\cdots+r^{m-1}\right)\left(\frac{1}{M\left(x_{0}, x_{1}, t\right)}-1\right) \\
& \leq \frac{r^{n}}{1-r}\left(\frac{1}{M\left(x_{0}, x_{1}, t\right)}-1\right),
\end{aligned}
$$

for all $m>n$. Therefore, $\left\{x_{n}\right\}$ is a Cauchy sequence. The completeness of $X$ ensures that the sequence $\left\{x_{n}\right\}$ converges to some $x^{*} \in X$; that is $\lim _{n \rightarrow+\infty} M\left(x_{n}, x^{*}, t\right)=1$, for all $t>0$.

Suppose there exists $n_{0} \in \mathbb{N}$ such that

$$
\begin{aligned}
& \frac{1}{1+r}\left(\frac{1}{M\left(x_{n_{0}-1}, T x_{n_{0}-1}, t\right)}-1\right) \\
& >\frac{1}{M\left(x_{n_{0}-1}, x^{*}, t\right)}-1, \\
& \frac{1}{1+r}\left(\frac{1}{M\left(x_{n_{0}}, T x_{n_{0}}, t\right)}-1\right) \\
& >\frac{1}{M\left(x_{n_{0}}, x^{*}, t\right)}-1 .
\end{aligned}
$$

Therefore by (92) we deduce

$$
\begin{aligned}
\frac{1}{M\left(x_{n_{0}-1}, T x_{n_{0}-1}, t\right)}-1 \\
=\frac{1}{M\left(x_{n_{0}-1}, x_{n_{0}}, t\right)}-1 \\
\leq \frac{1}{M\left(x_{n_{0}-1}, x, t\right)}-1+\frac{1}{M\left(x_{n_{0}}, x, t\right)}-1 \\
<\frac{1}{1+r}\left(\frac{1}{M\left(x_{n_{0}-1}, T x_{n_{0}-1}, t\right)}-1\right) \\
\quad+\frac{1}{1+r}\left(\frac{1}{M\left(x_{n_{0}}, T x_{n_{0}}, t\right)}-1\right)
\end{aligned}
$$

$$
\begin{aligned}
\leq & \frac{1}{1+r}\left(\frac{1}{M\left(x_{n_{0}-1}, T x_{n_{0}-1}, t\right)}-1\right) \\
& +\frac{r}{1+r}\left(\frac{1}{M\left(x_{n_{0}-1}, T x_{n_{0}-1}, t\right)}-1\right) \\
= & \frac{1}{M\left(x_{n_{0}-1}, T x_{n_{0}-1}, t\right)}-1,
\end{aligned}
$$

which is a contradiction. Hence, either

$$
\begin{aligned}
& \frac{1}{1+r}\left(\frac{1}{M\left(x_{n-1}, T x_{n-1}, t\right)}-1\right) \\
& \quad \leq \frac{1}{M\left(x_{n-1}, x^{*}, t\right)}-1
\end{aligned}
$$

or

$$
\frac{1}{1+r}\left(\frac{1}{M\left(x_{n}, T x_{n}, t\right)}-1\right) \leq \frac{1}{M\left(x_{n}, x^{*}, t\right)}-1
$$

holds for all $n \in \mathbb{N}$. So from (88) we get

$$
\begin{aligned}
& \frac{1}{M\left(x_{n}, T x^{*}, t\right)} \\
& \quad=\frac{1}{M\left(T x_{n-1}, T x^{*}, t\right)} \\
& \quad \leq \frac{r}{M\left(x_{n-1}, x^{*}, t\right)}+(1-r) M\left(x^{*}, T x_{n-1}, t\right) \\
& \quad \leq \frac{r}{M\left(x_{n-1}, x^{*}, t\right)}+(1-r) M\left(x^{*}, x_{n}, t\right)
\end{aligned}
$$

or

$$
\begin{aligned}
& \frac{1}{M}\left(x_{n+1}, T x^{*}, t\right) \\
& \quad=\frac{1}{M\left(T x_{n}, T x^{*}, t\right)} \\
& \quad \leq \frac{r}{M\left(x_{n}, x^{*}, t\right)}+(1-r) M\left(x^{*}, T x_{n}, t\right) \\
& \quad \leq \frac{r}{M\left(x_{n}, x^{*}, t\right)}+(1-r) M\left(x^{*}, x_{n+1}, t\right) .
\end{aligned}
$$

If we take limit as $n \rightarrow+\infty$ in each of the above inequalities, we obtain

$$
\frac{1}{M\left(x^{*}, T x^{*}, t\right)} \leq r+1-r=1 .
$$

This implies, $M\left(x^{*}, T x^{*}, t\right)=1$ for all $t>0$; that is, $x^{*}=$ $T x^{*}$. Finally, we show that $x^{*}$ is the unique fixed point of $T$. Assume, to the contrary, that $w \neq x^{*}$ is another fixed point of $T$. By Definition 1 , there exists $t_{0}>0$ such that $0 \leq$ $M\left(x^{*}, w, t_{0}\right)<1$. Then

$$
\frac{1}{1+r}\left(\frac{1}{M\left(x^{*}, T x^{*}, t_{0}\right)}-1\right)=0 \leq \frac{1}{M\left(x^{*}, w, t_{0}\right)}-1,
$$


and so by (88) we deduce

$$
\begin{aligned}
\frac{1}{M\left(T x^{*}, T w, t_{0}\right)} \leq & \frac{r}{M\left(x^{*}, w, t_{0}\right)} \\
& +(1-r) M\left(w, T x^{*}, t_{0}\right),
\end{aligned}
$$

which implies

$$
\frac{1-r}{M\left(x^{*}, w, t_{0}\right)} \leq(1-r) M\left(w, x^{*}, t_{0}\right) .
$$

That is, $1 \leq M\left(w, x^{*}, t_{0}\right)^{2}$. So, $M\left(w, x^{*}, t_{0}\right)=1$ which is a contradiction. Thus $T$ has a unique fixed point.

Example 25. Consider the space $X=\{(0,0),(0,4)$, $(4,0),(4,5),(5,4)\}$ endowed with the metric $d: X \times X \rightarrow$ $[0,+\infty)$ given by

$$
d\left(\left(x_{1}, x_{2}\right),\left(y_{1}, y_{2}\right)\right)=\left|x_{1}-y_{1}\right|+\left|x_{2}-y_{2}\right|
$$

for all $\left(x_{1}, x_{2}\right),\left(y_{1}, y_{2}\right) \in X$. Define intuitionistic fuzzy metric by

$$
\begin{array}{r}
M\left(\left(x_{1}, x_{2}\right),\left(y_{1}, y_{2}\right), t\right) \\
=\frac{1}{1+d\left(\left(x_{1}, x_{2}\right),\left(y_{1}, y_{2}\right)\right)}, \\
N\left(\left(x_{1}, x_{2}\right),\left(y_{1}, y_{2}\right), t\right) \\
\quad=\frac{d\left(\left(x_{1}, x_{2}\right),\left(y_{1}, y_{2}\right)\right)}{1+d\left(\left(x_{1}, x_{2}\right),\left(y_{1}, y_{2}\right)\right)},
\end{array}
$$

where $a * b=\min \{a, b\}$ and $a \diamond b=\max \{a, b\}$. Then $X$ is a complete triangular intuitionistic fuzzy metric space. Also define $T: X \rightarrow X$ by

$$
T\left(x_{1}, x_{2}\right)= \begin{cases}\left(x_{1}, 0\right) & \text { if } x_{1} \leq x_{2} \\ \left(0, x_{2}\right) & \text { if } x_{1}>x_{2}\end{cases}
$$

Now, we consider the following cases.

(i) If $(x, y)=((0,0),(0,4))$, then

$$
\begin{aligned}
\frac{1}{M(T(0,0), T(0,4), t)}= & 1 \leq \frac{0.9}{M((0,0),(0,4), t)} \\
& +(1-0.9) M((0,4), T(0,0), t) .
\end{aligned}
$$

(ii) If $(x, y)=((0,4),(0,0))$, then

$$
\begin{aligned}
\frac{1}{M(T(0,4), T(0,0), t)}= & 1 \leq \frac{0.9}{M((0,4),(0,0), t)} \\
& +(1-0.9) M(T(0,0),(0,4), t) .
\end{aligned}
$$

(iii) If $(x, y)=((0,0),(4,0))$, then

$$
\begin{aligned}
\frac{1}{M(T(0,0), T(4,0), t)}= & 1 \leq \frac{0.9}{M((0,0),(4,0), t)} \\
& +(1-0.9) M((4,0), T(0,0), t) .
\end{aligned}
$$

(iv) If $(x, y)=((0,0),(4,5))$, then

$$
\begin{aligned}
\frac{1}{M(T(0,0), T(4,5), t)}= & 5 \leq \frac{0.9}{M((0,0),(4,5), t)} \\
& +(1-0.9) M((4,5), T(0,0), t) .
\end{aligned}
$$

(v) If $(x, y)=((4,5),(0,0))$, then

$$
\begin{aligned}
\frac{1}{M(T(4,5), T(0,0), t)}= & 5 \leq \frac{0.9}{M((4,5),(0,0), t)} \\
& +(1-0.9) M(T(0,0),(4,5), t) .
\end{aligned}
$$

(vi) If $(x, y)=((0,0),(5,4))$, then

$$
\begin{aligned}
\frac{1}{M(T(0,0), T(5,4), t)}= & 5 \leq \frac{0.9}{M((0,0),(5,4), t)} \\
& +(1-0.9) M((5,4), T(0,0), t) .
\end{aligned}
$$

(vii) If $(x, y)=((5,4),(0,0))$, then

$$
\begin{aligned}
\frac{1}{M(T(5,4), T(0,0), t)}= & 5 \leq \frac{0.9}{M((5,4),(0,0), t)} \\
& +(1-0.9) M((0,0), T(5,4), t) .
\end{aligned}
$$

(viii) If $(x, y)=((0,4),(4,0))$, then

$$
\begin{aligned}
\frac{1}{M(T(0,4), T(4,0), t)}= & 1 \leq \frac{0.9}{M((0,4),(4,0), t)} \\
& +(1-0.9) M((4,0), T(0,4), t) .
\end{aligned}
$$

(ix) If $(x, y)=((4,0),(0,4))$, then

$$
\begin{aligned}
\frac{1}{M(T(4,0), T(0,4), t)}= & 1 \leq \frac{0.9}{M((4,0),(0,4), t)} \\
& +(1-0.9) M((0,4), T(4,0), t) .
\end{aligned}
$$

(x) If $(x, y)=((0,4),(4,5))$, then

$$
\begin{aligned}
\frac{1}{M(T(0,4), T(4,5), t)}= & 5 \leq \frac{0.9}{M((0,4),(4,5), t)} \\
& +(1-0.9) M((4,5), T(0,4), t) .
\end{aligned}
$$

(xi) If $(x, y)=((4,5),(0,4))$, then

$$
\begin{aligned}
\frac{1}{M(T(4,5), T(0,4), t)}= & 5 \leq \frac{0.9}{M((4,5),(0,4), t)} \\
& +(1-0.9) M((0,4), T(4,5), t) .
\end{aligned}
$$


(xii) If $(x, y)=((0,4),(5,4))$, then

$$
\begin{aligned}
\frac{1}{M(T(0,4), T(5,4), t)}= & 5 \leq \frac{0.9}{M((0,4),(5,4), t)} \\
& +(1-0.9) M((5,4), T(0,4), t) .
\end{aligned}
$$

(xiii) If $(x, y)=((5,4),(0,4))$, then

$$
\begin{aligned}
\frac{1}{M(T(5,4), T(0,4), t)}= & 5 \leq \frac{0.9}{M((5,4),(0,4), t)} \\
& +(1-0.9) M((0,4), T(5,4), t) .
\end{aligned}
$$

(xiv) If $(x, y)=((4,5),(5,4))$, then

$$
\begin{aligned}
\frac{1}{M(T(4,5), T(5,4), t)}= & 9>\frac{0.9}{M((4,5),(5,4), t)} \\
& +(1-0.9) M((4,5), T(5,4), t) .
\end{aligned}
$$

Also notice that

$$
\begin{gathered}
\frac{1}{1+0.9}\left(\frac{1}{M((4,5), T(4,5), t)}-1\right) \\
>\frac{1}{M((4,5),(5,4), t)}-1 .
\end{gathered}
$$

$(\mathrm{xv})$ If $(x, y)=((5,4),(4,5))$, then

$$
\begin{aligned}
\frac{1}{M(T(5,4), T(4,5), t)}= & 9>\frac{0.9}{M((5,4),(4,5), t)} \\
& +(1-0.9) M((5,4), T(4,5), t) .
\end{aligned}
$$

But note that

$$
\begin{gathered}
\frac{1}{1+0.9}\left(\frac{1}{M((5,4), T(5,4), t)}-1\right) \\
>\frac{1}{M((5,4),(4,5), t)}-1
\end{gathered}
$$

Consequently, we obtain

$$
\begin{aligned}
& \frac{1}{1+0.9}\left(\frac{1}{M(x, T x, t)}-1\right) \\
& \quad \leq \frac{1}{M(x, y, t)}-1 \Longrightarrow \frac{1}{M(T x, T y, t)} \\
& \quad \leq \frac{0.9}{M(x, y, t)}+(1-0.9) M(y, T x, t) .
\end{aligned}
$$

Hence, all conditions of Theorem 24 hold and $T$ has $(0,0)$ as a unique fixed point.

From Theorem 24, we deducethe following corollary.
Corollary 26. Let $(X, M, N, *, \diamond)$ be a complete triangular intuitionistic fuzzy metric space and let $T$ be a self-mapping on $X$. Assume that there exists $r \in(0,1)$ such that

$$
\frac{1}{M(T x, T y, t)} \leq \frac{r}{M(x, y, t)}+(1-r) M(y, T x, t),
$$

for all $x, y \in X$ and all $t>0$. Then $T$ has a unique fixed point.

\section{Conflict of Interests}

The authors declare that there is no conflict of interests regarding the publication of this paper.

\section{Acknowledgments}

This paper was funded by the Deanship of Scientific Research (DSR), King Abdulaziz University, Jeddah. The first and fourth authors acknowledge with thanks DSR, KAU, for financial support. The authors would also like to thank the editor and referees for their constructive comments which considerably improved the paper.

\section{References}

[1] L. A. Zadeh, "Fuzzy sets," Information and Computation, vol. 8, pp. 338-353, 1965.

[2] I. Kramosil and J. Michálek, "Fuzzy metrics and statistical metric spaces," Kybernetika, vol. 11, no. 5, pp. 336-344, 1975.

[3] M. Grabiec, "Fixed points in fuzzy metric spaces," Fuzzy Sets and Systems, vol. 27, no. 3, pp. 385-389, 1988.

[4] A. George and P. Veeramani, "On some results in fuzzy metric spaces," Fuzzy Sets and Systems, vol. 64, no. 3, pp. 395-399, 1994.

[5] C. Di Bari and C. Vetro, "Fixed points, attractors and weak fuzzy contractive mappings in a fuzzy metric space," Journal of Fuzzy Mathematics, vol. 13, no. 4, pp. 973-982, 2005.

[6] D. Gopal, M. Imdad, C. Vetro, and M. Hasan, "Fixed point theory for cyclic weak $\phi$-contraction in fuzzy metric spaces," Journal of Nonlinear Analysis and Application, vol. 2012, Article ID jnaa-00110, 11 pages, 2012.

[7] P. Salimi, C. Vetro, and P. Vetro, "Some new fixed point results in non-Archimedean fuzzy metric spaces," Nonlinear Analysis: Modelling and Control, vol. 18, no. 3, pp. 344-358, 2013.

[8] Y. Shen, D. Qiu, and W. Chen, "Fixed point theorems in fuzzy metric spaces," Applied Mathematics Letters, vol. 25, no. 2, pp. 138-141, 2012.

[9] C. Vetro, "Fixed points in weak non-Archimedean fuzzy metric spaces," Fuzzy Sets and Systems, vol. 162, pp. 84-90, 2011.

[10] C. Vetro, D. Gopal, and M. Imdad, "Common fixed point theorems for $(\phi, \psi)$-weak contractions in fuzzy metric spaces," Indian Journal of Mathematics, vol. 52, no. 3, pp. 573-590, 2010.

[11] C. Vetro and P. Vetro, "Common fixed points for discontinuous mappings in fuzzy metric spaces," Rendiconti del Circolo Matematico di Palermo, vol. 57, no. 2, pp. 295-303, 2008.

[12] J. H. Park, "Intuitionistic fuzzy metric spaces," Chaos, Solitons and Fractals, vol. 22, no. 5, pp. 1039-1046, 2004.

[13] C. Alaca, D. Turkoglu, and C. Yildiz, "Fixed points in intuitionistic fuzzy metric spaces," Chaos, Solitons \& Fractals, vol. 29, no. 5, pp. 1073-1078, 2006. 
[14] D. Çoker, "An introduction to intuitionistic fuzzy topological spaces," Fuzzy Sets and Systems, vol. 88, no. 1, pp. 81-89, 1997.

[15] J. S. Park, Y. C. Kwun, and J. H. Park, "A fixed point theorem in the intuitionistic fuzzy metric spaces," Far East Journal of Mathematical Sciences, vol. 16, no. 2, pp. 137-149, 2005.

[16] M. Rafi and M. S. M. Noorani, "Fixed point theorem on intuitionistic fuzzy metric spaces," Iranian Journal of Fuzzy Systems, vol. 3, no. 1, pp. 23-29, 2006.

[17] B. Schweizer and A. Sklar, "Statistical metric spaces," Pacific Journal of Mathematics, vol. 10, pp. 313-334, 1960.

[18] C. Di Bari and C. Vetro, "A fixed point theorem for a family of mappings in a fuzzy metric space," Rendiconti del Circolo Matematico di Palermo II, vol. 52, no. 2, pp. 315-321, 2003.

[19] C. Ionescu, S. Rezapour, and M. E. Samei, "Fixed points of some new contractions on intuitionistic fuzzy metric spaces," Fixed Point Theory and Applications, vol. 2013, article 168, 2013.

[20] M. A. Alghamdi, N. Hussain, and P. Salimi, "Fixed point and coupled fixed point theorems on b-metric-like spaces," Journal of Inequalities and Applications, vol. 2013, article 402, 2013.

[21] D. Paesano and P. Vetro, "Suzuki's type characterizations of completeness for partial metric spaces and fixed points for partially ordered metric spaces," Topology and Its Applications, vol. 159, no. 3, pp. 911-920, 2012.

[22] P. Salimi and E. Karapınar, "Suzuki-Edelstein type contractions via auxiliary functions," Mathematical Problems in Engineering, Article ID 648528, 8 pages, 2013.

[23] T. Suzuki, "A new type of fixed point theorem in metric spaces," Nonlinear Analysis: Theory, Methods \& Applications, vol. 71, no. 11, pp. 5313-5317, 2009.

[24] T. Suzuki, "A generalized Banach contraction principle that characterizes metric completeness," Proceedings of the American Mathematical Society, vol. 136, no. 5, pp. 1861-1869, 2008.

[25] W. Takahashi, N.-C. Wong, and J.-C. Yao, "Fixed point theorems for general contractive mappings with $W$-distances in metric spaces," Journal of Nonlinear and Convex Analysis, vol. 14, no. 3 , pp. 637-648, 2013.

[26] N. Hussain, D. Đorić, Z. Kadelburg, and S. Radenović, "Suzukitype fixed point results in metric type spaces," Fixed Point Theory and Applications, vol. 2012, article 126, 2012.

[27] N. Hussain A Latif and P. Salimi, "Best proximity point results for modified Suzuk $\alpha$ - $\psi$-proximal contractions," Fixed Point Theory and Applications, vol. 2014, article 10, 2014.

[28] N. Shobkolaei, S. Sedghi, J. R. Roshan, and N. Hussain, "Suzukitype fixed point results in metric-like spaces," Journal of Function Spaces and Applications, vol. 2013, Article ID 143686, 9 pages, 2013.

[29] N. Hussain, M. A. Kutbi, S. Khaleghizadeh, and P. Salimi, "Discussions on recent results for $\alpha-\psi$-contractive mappings," Abstract and Applied Analysis, vol. 2014, Article ID 456482, 13 pages, 2014. 


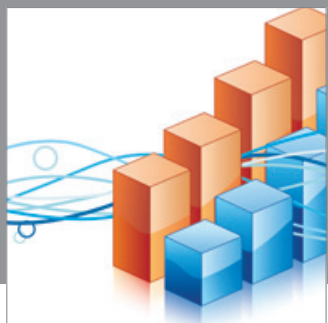

Advances in

Operations Research

mansans

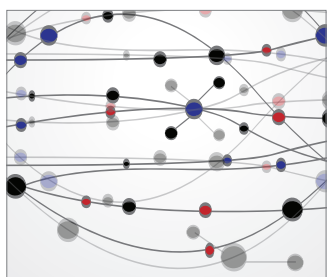

The Scientific World Journal
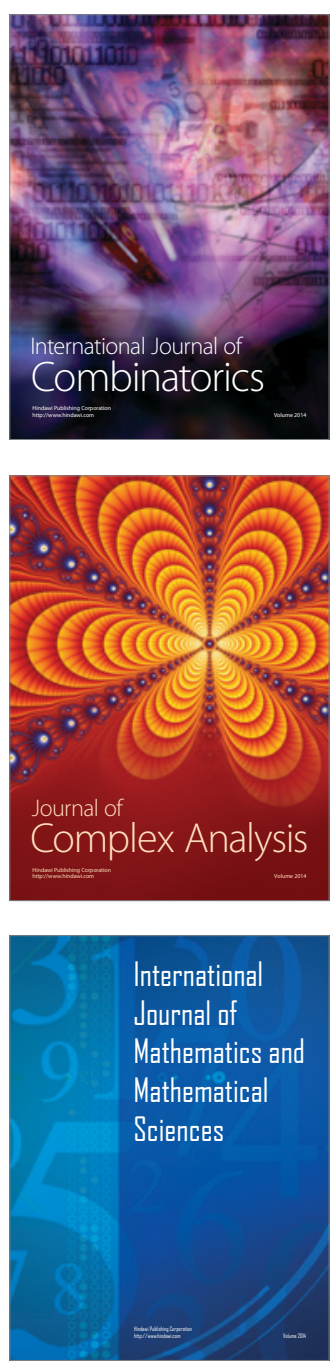
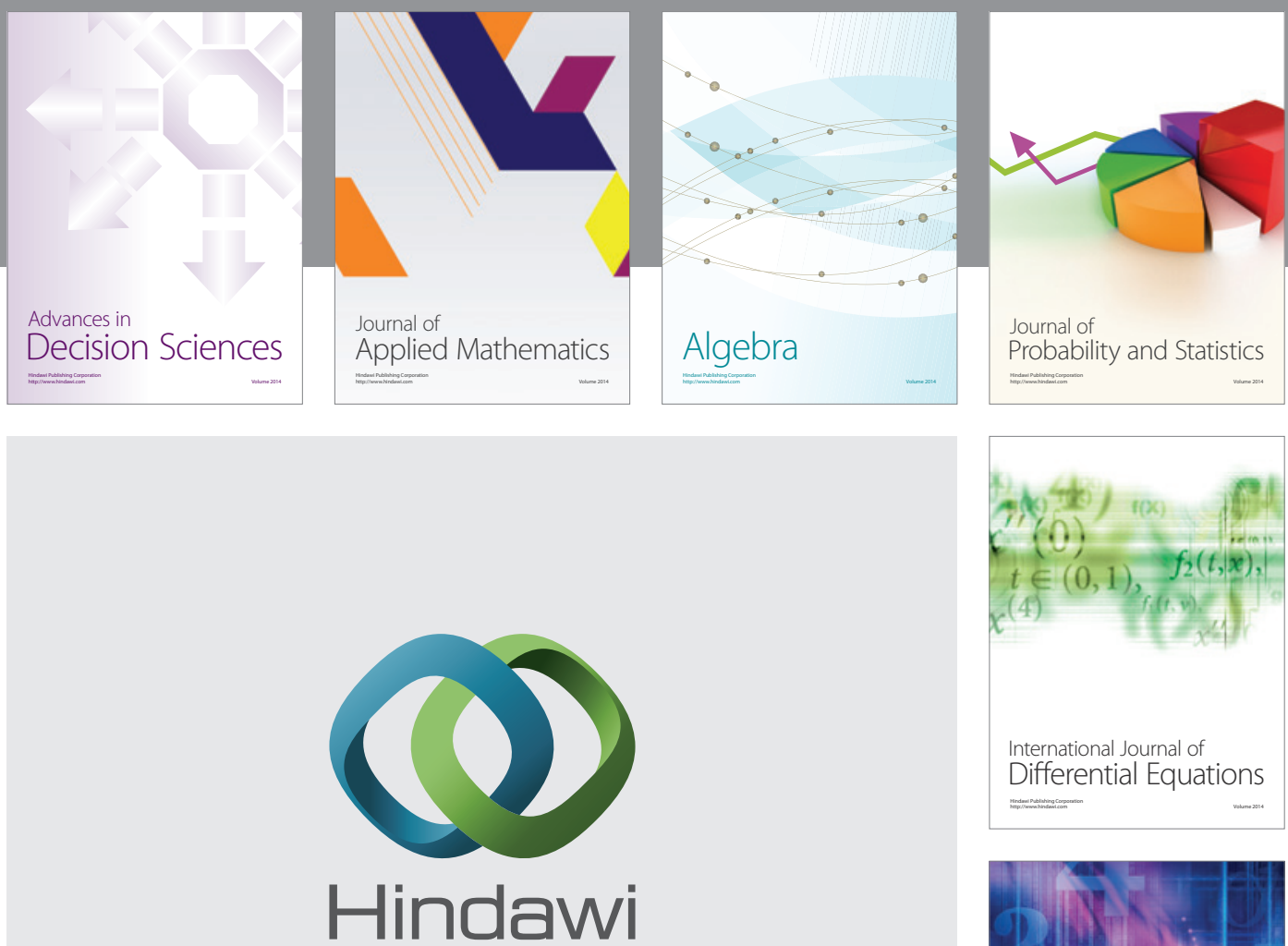

Submit your manuscripts at http://www.hindawi.com
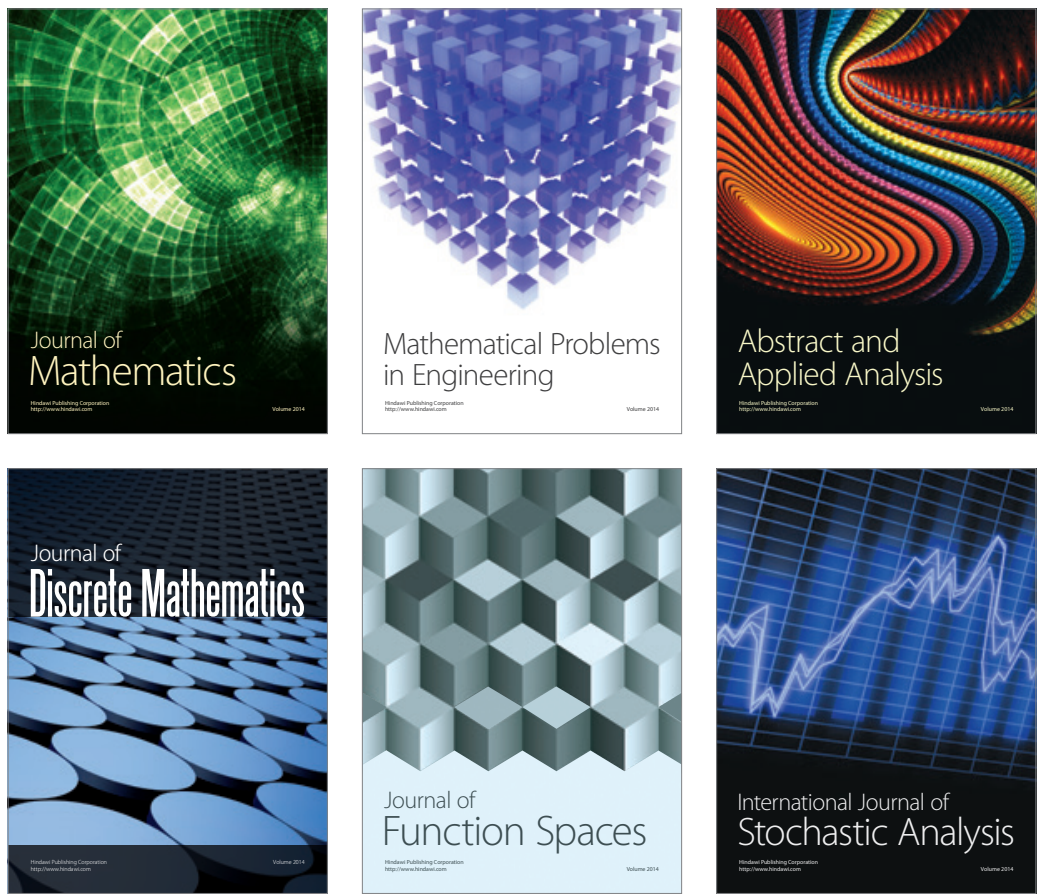

Journal of

Function Spaces

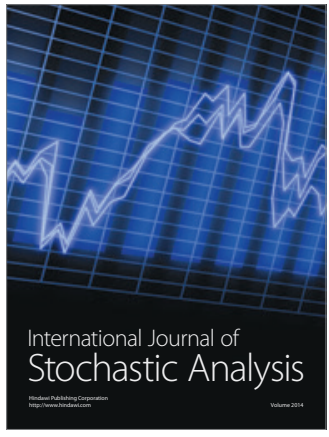

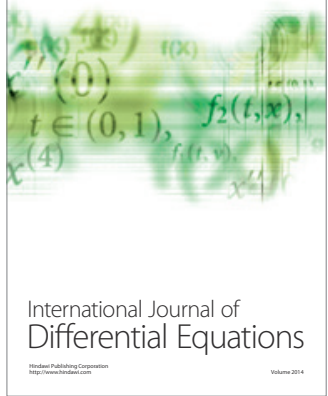
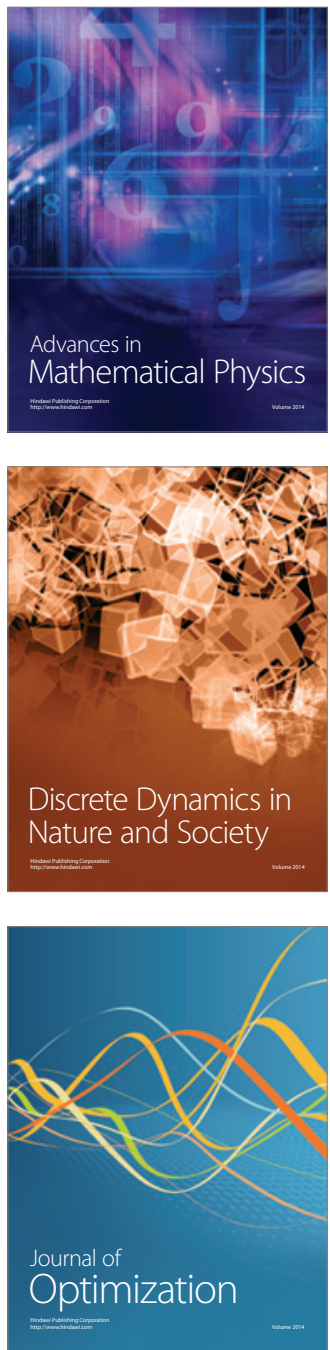